\title{
1 Delta oscillations coordinate intra-cerebellar and cerebello-hippocampal network 2 dynamics during sleep
}

3 Torres-Herraez $\mathrm{A}^{1}$, Watson TC*1 and Rondi-Reig $\mathrm{L}^{* 15}$

$4 \quad{ }^{1}$ Sorbonne Université, CNRS, INSERM, Institut de Biologie Paris Seine (IBPS), Neurosciences Paris Seine

5 (NPS), Cerebellum Navigation and Memory Team (CeZaMe), F-75005 Paris, France

6 ATH current address: Department of Psychiatry, Columbia University Medical Center, New York, NY $7 \quad 10032$

8 TCW current address: Centre for Discovery Brain Sciences, University of Edinburgh, Edinburgh, United 9 Kingdom; Simons Initiative for the Developing Brain, University of Edinburgh, Edinburgh, United 10 Kingdom; Patrick Wild Centre for Autism Research, University of Edinburgh, Edinburgh, United 11 Kingdom

$12 \S$ Corresponding author: laure.rondi-reig@sorbonne-universite.fr

13 IBPS, Neurosciences Paris Seine, Sorbonne Université, Campus Jussieu, CeZaMe team, 9 Quai Saint 14 Bernard, 5e étage, Pièce 519, 75005 Paris, France

$15 *$ These authors contributed equally to this work 


\section{Abstract:}

During sleep, the widespread coordination of neuronal oscillations across both cortical and subcortical brain regions is thought to support various physiological functions, including memory consolidation. However, how sleep-related activity within the brain's largest sensorimotor structure, the cerebellum, is multiplexed with well described sleep-related mechanisms in regions such as the hippocampus remains unknown.

To address this gap in knowledge, we simultaneously recorded from the dorsal hippocampus and three distinct regions of the cerebellum (Crus I, lobule VI and lobules II/III) during natural murine sleep. We found that LFP oscillations are coordinated between the two structures in a sleep-stage specific manner. Particularly during non-REM sleep, prominent delta frequency coherence was observed between lobule VI and hippocampus. We additionally observed that non-REM associated hippocampal sharp wave ripple activity can drive discrete LFP modulation in all the recorded cerebellar regions, with the shortest latency effects observed in lobule VI.

We also describe discrete phasic sharp potentials, synchronized across cerebellar regions, which were strongly phase locked to the peak of ongoing cerebellar delta oscillations and found in greatest numbers during REM. These phasic sharp potentials recorded within the cerebellar cortex were found to be phase-locked to the trough of the hippocampal theta oscillation, further suggesting crossstructural coordination. During REM, cerebellar delta oscillation phase significantly modulated hippocampal theta frequency, and this effect was greatest when phasic sharp potentials were most abundant. Within all three cerebellar regions, prominent LFP oscillations were observed at both low (delta, $<4 \mathrm{~Hz}$ ) and very high frequencies $(\sim 250 \mathrm{~Hz}$ ) during non-REM and REM sleep. Intra-cerebellar cross-frequency analysis revealed that delta frequency oscillations strongly modulate those in the very high frequency range.

Together, these results reveal multiple candidate physiological mechanisms to support 'offline', bidirectional interaction within distributed cerebello-hippocampal networks. In particular, we describe a prominent cerebellar delta oscillation, which appears to act as a temporal coordinator of cerebellar activity at both local (intra-cerebellar) and distributed (cerebello-hippocampal) network levels. 
60

61

\section{Introduction}

Many important physiological processes are associated with sleep, including memory formation and consolidation (Diekelmann and Born, 2010). In particular, sleep-related neurophysiological events in the hippocampus, such as place cell reactivations and high frequency sharp wave ripples are vital to spatial memory formation (e.g. de Lavilléon et al., 2015). Additionally, widespread slow wave oscillations are thought to play a crucial role in the temporal coordination of discrete sleep-related events, such as hippocampal ripples and thalamo-cortical spindles, across a large network of brain regions (e.g. Nicola et al., 2019)

Recent studies have shown that during wakeful behaviour the cerebellum is crucial for hippocampal place cell stability and efficient navigation (Rochefort et al., 2011; Lefort et al., 2019; Burguière et al., 2005, Babayan et al., 2017). Furthermore, the neuroanatomical connectivity required to support this influence has been recently described and physiological correlates of cerebello-hippocampal interaction identified 'online' during goal-directed navigation (Watson et al., 2019). So far, however, nothing is known about possible 'offline' cerebello-hippocampal physiological interactions during sleep.

To date, sleep research has primarily focused on state-dependent correlation in neocortical and subcortical structures. However, early studies in epileptic patients implanted with intra-cerebellar electrodes (Niedermeyer and Uematsu, 1974) and recent work using non-invasive techniques (Jahnke et al., 2012; Kaufmann et al., 2006; Schabus et al., 2007) have described state-dependent modulation of cerebellar activity during sleep in humans. During non-rapid eye movement (non-REM) sleep, coordinated slow oscillations, recorded predominantly in cerebellar vermis and fastigial nucleus (Niedermeyer and Uematsu, 1974; Schabus et al., 2007), are correlated with neocortical K-complexes (Jahnke et al., 2012) and sleep spindles (Schabus et al., 2007). During REM sleep, an increase in bloodoxygen-level-dependent signals has been observed in both the cerebellar vermis and hemispheres (Braun, 1997).

Similarly, single-cell recording in animal models has revealed sleep-state related changes in both Purkinje cell and deep cerebellar nuclei neuron firing. During non-REM, no clear neuronal firing rate changes were observed either in cats (Hobson and McCarley, 1972; Marchesi and Strata, 1970; McCarley and Hobson, 1972; Palmer, 1979) or monkeys (Mano, 1970). However, a reduced probability for short interspike intervals was observed in Purkinje cell simple spike firing (McCarley and Hobson, 1972). Consistent with observations made in humans, during REM sleep a significant increase in the firing rate of Purkinje cells was observed in the cerebellar cortex of cats (Hobson and McCarley, 1972; Marchesi and Strata, 1970) and monkeys (Mano, 1970). The presence of large amplitude, phasic 
events in the cerebellum of cats (Harlay et al., 1974; Pellet and Harley, 1977) and rats (Marks et al., 1980), mainly during REM sleep epochs, has also been attributed to the transmission of pontogeniculo-occipital waves (PGO waves; Farber et al., 1980; Velluti et al., 1985). Finally, recent studies have highlighted cerebellar roles in the generation of sleep spindles in monkeys (Xu et al., 2020) and sleep-wake transitions in mice (Zhang et al., 2020).

Thus, in addition to the multitude of studies highlighting the functional importance of sleep-state dependent processes in the hippocampus (see Klinzing et al., 2019 for review) the aforementioned evidence indicates clear cerebellar activity modulation during sleep (reviewed in Canto et al., 2017). Nevertheless, our understanding of spatial and temporal organisation of cerebellar activity across sleep states remains rudimentary. Furthermore, to the best of our knowledge, nothing is known about the physiological links between cerebellum and hippocampus during sleep. We therefore simultaneously recorded spontaneous local field potentials (LFPs) from the dorsal hippocampus, vermal cerebellar lobules II/III (Lob II/III), VI (Lob VI) and hemispheric Crus I in freely behaving and sleeping mice. Our analysis reveals that delta oscillations, along with associated phasic sharp potentials (PSPs), coordinate both local cerebellar and distributed cerebello-hippocampal network dynamics in a sleep-state dependent manner.

\section{$\underline{\text { Results }}$}

\section{Spectral profiling of cerebellar and hippocampal activity across sleep states}

In line with previous studies (e.g. Montgomery et al., 2008), hippocampal LFP was dominated by sustained, low amplitude theta oscillations $(6-12 \mathrm{~Hz})$ during wake and REM states, and high amplitude slow oscillations in the delta range $(<4 \mathrm{~Hz}$ ) during non-REM sleep (Figure $1 \mathrm{~A}-\mathrm{C}$ ).

Conversely, high amplitude low frequency oscillations (including $<4 \mathrm{~Hz}$ delta waves) were characteristic of wake, REM and non-REM states in the cerebellum (Figure $1 \mathrm{~A}-\mathrm{C}$ ). However, differences in delta oscillation power, as well as peak frequency, were observed both across cerebellar subregions and wake/sleep states (Figure 1C, Supplemental Figure 1A-B). Similarly, a significant shift in the peak delta frequency was observed during non-REM sleep, specifically in Lob VI (Supplemental Figure 1B). In contrast, these differences were not observed during REM, when similar spectral profiles were found across cerebellar regions (Figure 1C). Moreover, delta oscillations were highly coherent between cerebellar recording sites (Figure 1B, Supplementary Figure 2), suggesting they may play a role in intra-cerebellar coordination during REM sleep. 
In addition to delta activity during wake, high-frequency oscillations $(100-160 \mathrm{~Hz})$ were also observed in the cerebellar power spectra (Figure 1C). However, during both non-REM and REM states, power in the high-frequency range was overshadowed by a prominent, narrowband $\sim 240-280 \mathrm{~Hz}$ oscillation (classified as very-high frequency oscillations, VHFOs; de Solages et al., 2008; Figure 1C; Supplemental Figure $1 C$ ). Local variability within this frequency band was observed across cerebellar regions and sleep states, with maximal values observed in Lob II/III during non-REM and REM sleep (Supplemental Figure 1D). In contrast, Crus I VHFO power was particularly reduced in REM (Figure 1C, Supplemental Figure 1D, paired t-tests: Crus I: $n=11, p=0.0005$; Lob II/III, $n=11$, Crus I, $n=11$; Lob VI, $n=15$; Lob II/III, $\mathrm{n}=11$ mice).

Overall, these data illustrate the presence of lobule and sleep-state dependent LFP dynamics across anterior (Lob II/III), posterior (Lob VI) and hemispheric (Crus I) cerebellum and highlight the existence of a prominent cerebellar delta oscillation.

\section{Cerebello-hippocampal coherence across sleep states}

Next, we sought to measure the level of functional coupling (measured as coherence; cf Watson et al., 2019; Xu et al., 2020) between the cerebellum and hippocampus during wake/sleep states (Figure 1D, E). During wake, coherence peaks were similarly observed across delta and theta frequencies in all cerebello-hippocampal combinations (Figure 1D, left panel). Strikingly, as mice moved to non-REM sleep, delta band coherence between hippocampus and Lob VI was significantly increased relative to other recorded cerebellar regions (Figure 1D, Supplemental Figure 3) indicating region-specific cerebello-hippocampal coupling during this sleep state. Furthermore, significant modulation of delta and theta coherence across sleep states was present in HPC-Lob VI and HPC-Lob II/III recording combinations, while remaining stable between HPC-Crus I (Figure 1D; Supplemental Figure 3). In contrast, during REM sleep, coherence patterns were similar to wake, with the most prominent peaks observed in the theta and low frequency range ( $<1 \mathrm{~Hz}$; Figure 1D, right panel; Supplemental Figure 3).

149 In summary, by characterizing the spectral properties of cerebello-hippocampal network LFP during wake and sleep we reveal that coherence between these regions is sleep-state dependent and particularly prominent between hippocampus and Lob VI during the non-REM stage of sleep. 


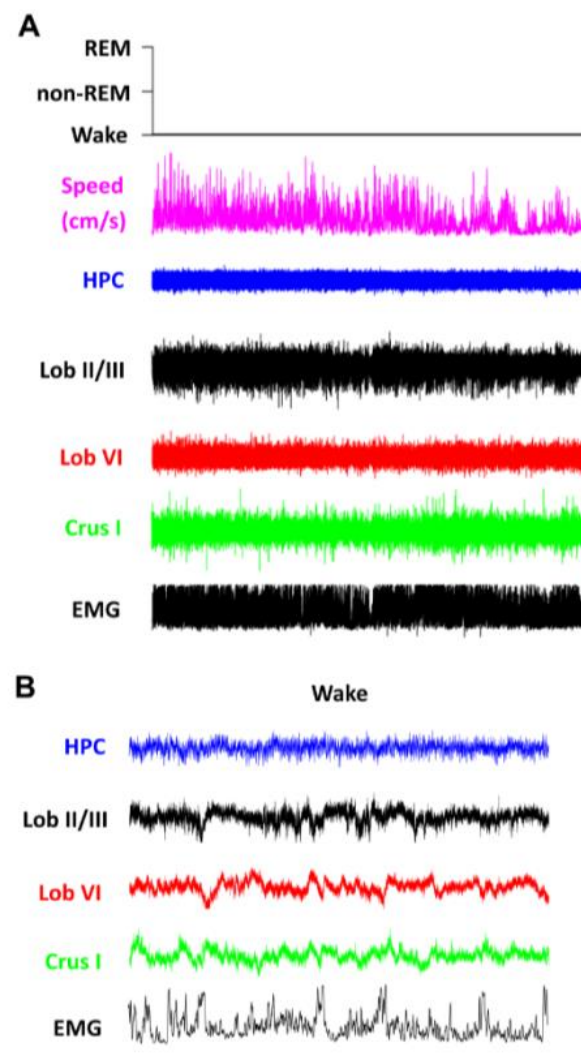

C

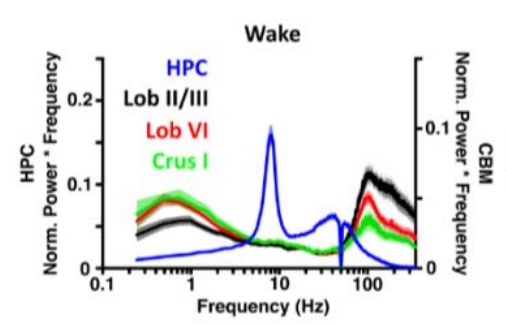

D
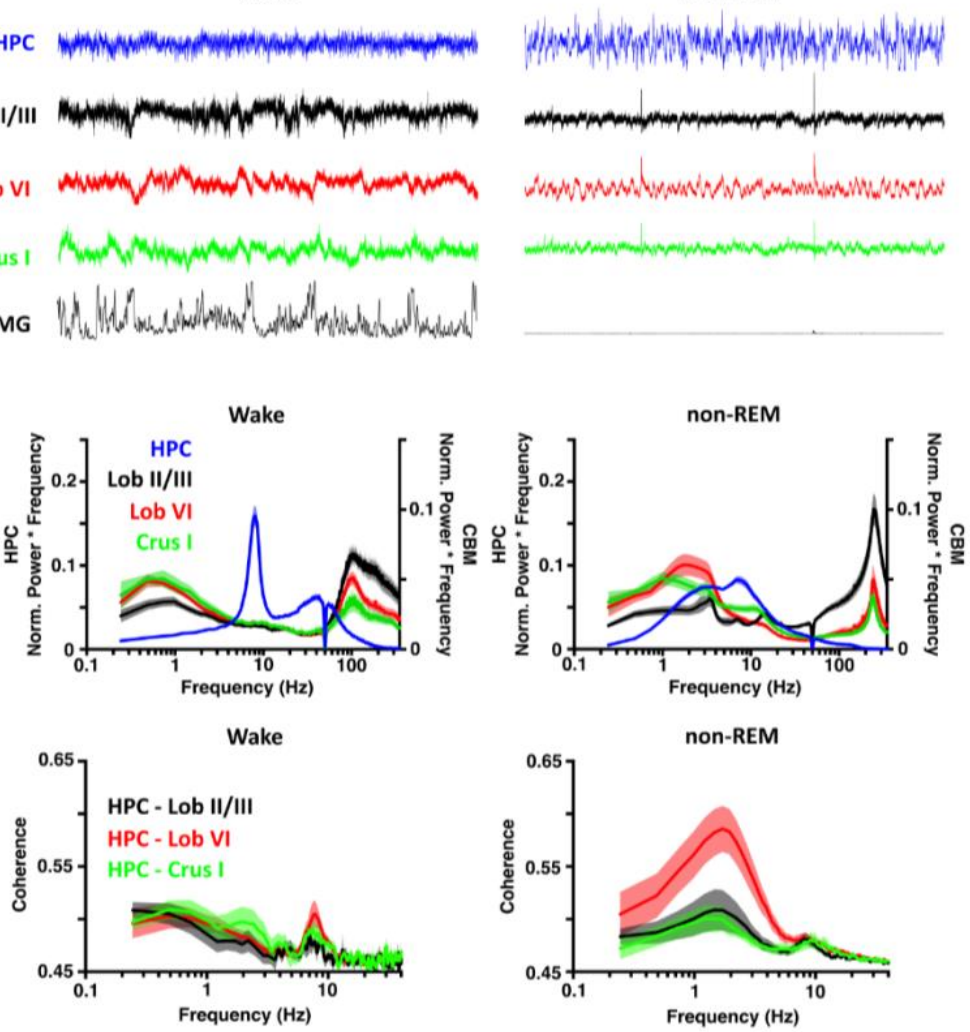

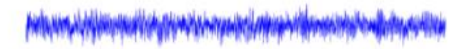

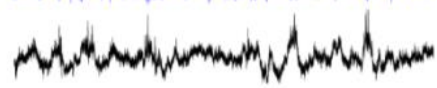

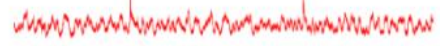

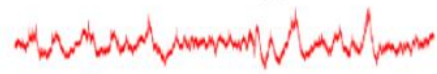

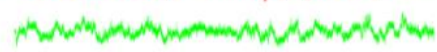

non-REM
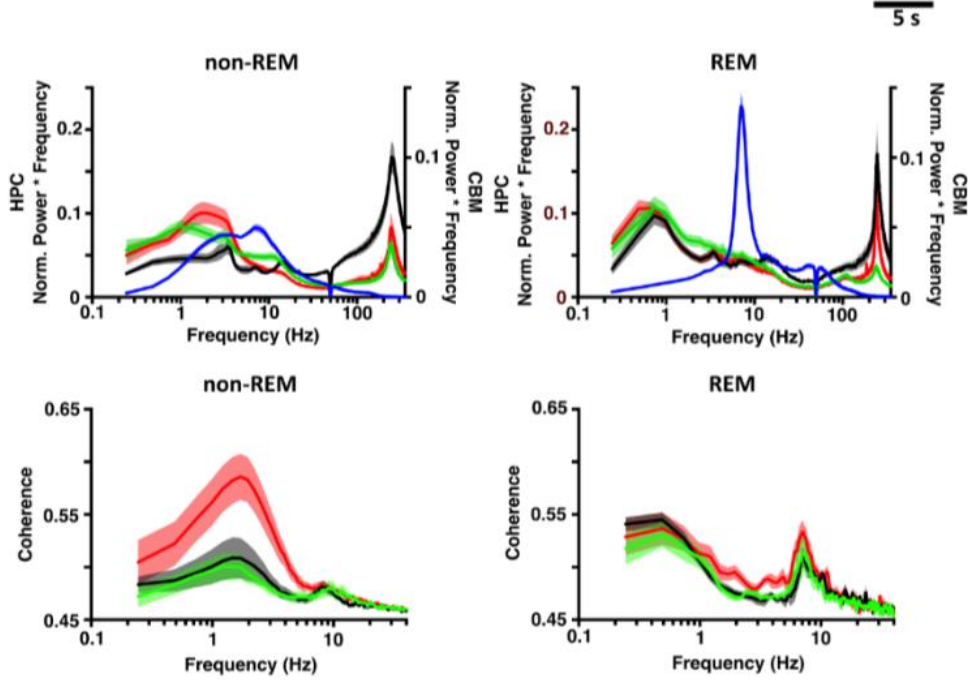

REM

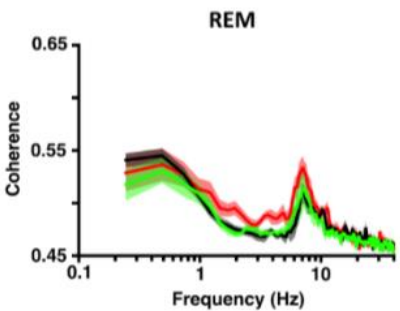

Figure 1. Sleep-state specific activity patterns are present in the cerebello-hippocampal network.

A, Hippocampal (HPC) and cerebellar cortical (Crus I, Lob VI and II/III) LFPs were recorded as mice cycled between defined wake, non-REM and REM states. B, During wake, hippocampal theta and cerebellar $<1 \mathrm{~Hz}$ and high gamma $(100-160 \mathrm{~Hz})$ oscillations occurred concomitantly. Similarly, during REM, hippocampal theta oscillations were accompanied by widespread delta $(<4 \mathrm{~Hz})$ and very fast ( $250 \mathrm{~Hz}$ ) cerebellar oscillations. During non-REM, high amplitude hippocampal activity co-occurred with both slow, phasic and very high frequency $(\sim 250 \mathrm{~Hz})$ cerebellar oscillations. C, Mean power spectra for each of the defined states (HPC, $n=20$; Crus I, $n=11$; Lob VI, $n=15$; Lob II/III, $n=11$ ). D, Mean coherence between HPC-Crus I $(n=11)$; HPC-Lob VI $(n=15)$ and HPC-Lob II/III $(n=11)$ across states. 
165 REM associated cerebellar phasic sharp potentials phase lock to both local cerebellar and distant

166 hippocampal oscillations, respectively.

167 In addition to the striking sleep-state related changes observed in ongoing cerebellar LFP oscillations,

168 we also noted prominent phasic sharp potential events (PSPs; large-amplitude voltage fluctuations of

$169129.5 \pm 5.68 \mathrm{~ms}$ duration and $2.31 \pm 0.15 \mathrm{z}$-score amplitude) across the three cerebellar recording sites

170 (Figure 2A-C). PSPs occurred in all three cerebellar regions at similar times (cross-correlation between

171 all pairs of cerebellar recorded PSPs revealed a peak at 0 latency; Figure $2 \mathrm{~A}-\mathrm{C}$ ) and were found during

172 both REM and non-REM epochs; however, they occurred in greatest numbers during REM (PSP density

173 during non-REM $=0.12 \pm 0.01$, PSP density during REM $=0.5 \pm 0.05$, Wilcoxon test $P<0.0001$; Figure

$1742 \mathrm{~A}, \mathrm{D})$. Moreover, PSPs occurred in concentrated clusters during REM (inter-event interval < 1s) but

175 were distributed more sparsely during non-REM (Figure 2E). We also observed that during REM

176 epochs, PSPs tended to occur at specific phases of the ongoing cerebellar delta waves in all three

177 cerebellar regions (Figure 2A). This observation was confirmed by computing the distribution of PSP

178 events relative to the phase of REM associated cerebellar delta waves (Figure 2F-H; PSPs occurred

179 most often near the peak of the cycle with a preferred angle of $350.6 \pm 2.248^{\circ}$ and with similar levels

180 across all three cerebellar regions, Figure 2G, H). Next, we investigated phase locking of PSPs recorded

181 in the cerebellum relative to hippocampal LFP theta phase during REM (Figure 2I), which revealed that

182 these events occur consistently near the trough of the theta cycle (preferred phase angle, $147.4 \pm$

$18315.93^{\circ}$; Figure $2 \mathrm{I}$ ) and at similar levels across cerebellar regions (Figure $2 \mathrm{~J}, \mathrm{~K}$ ). 
A

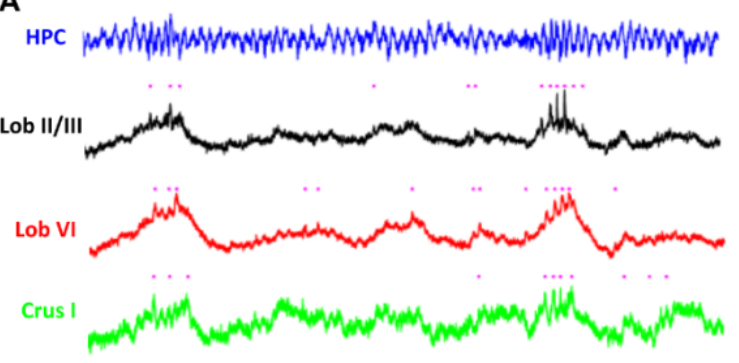

C

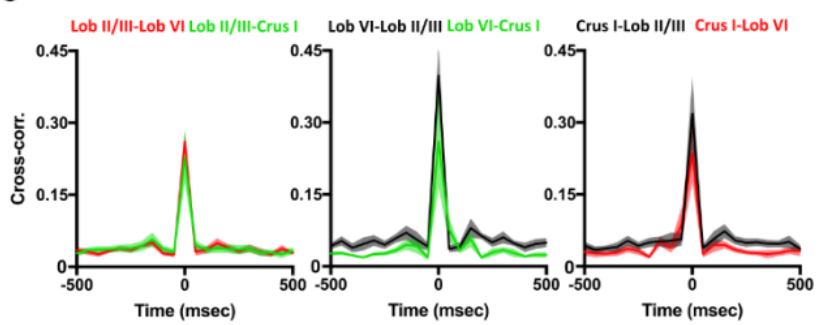

$\mathbf{F}$
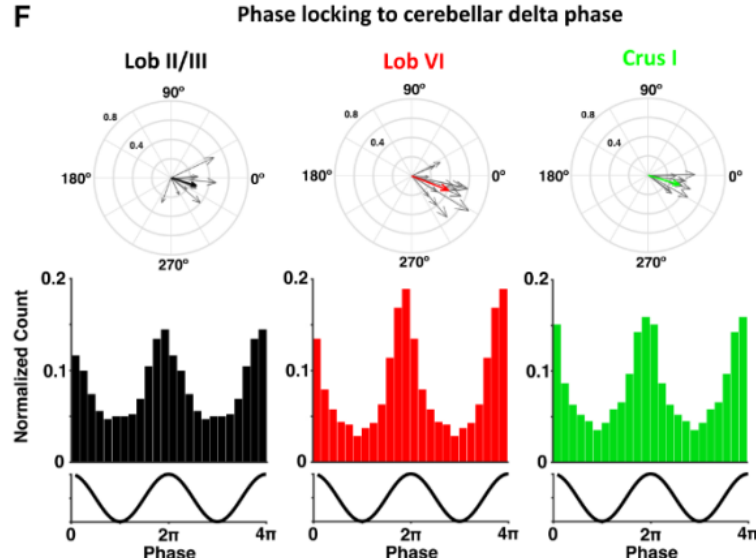

G
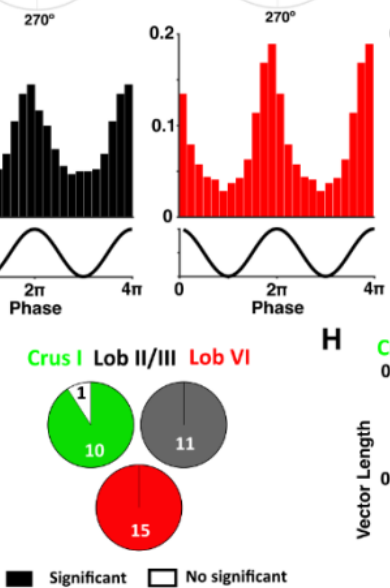

$\mathrm{H}$

$$
\text { H }
$$

I

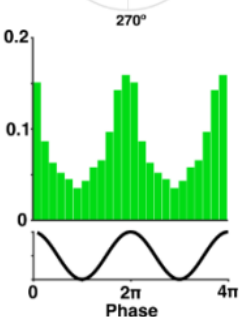

Crus I Lob II/III Lob VI

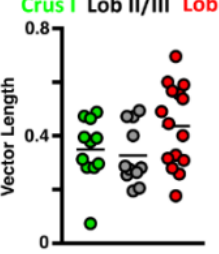

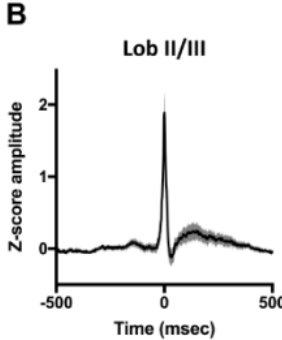
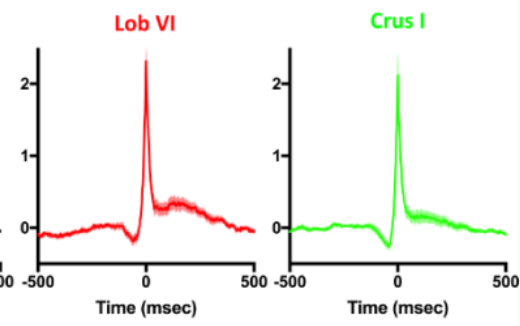

D

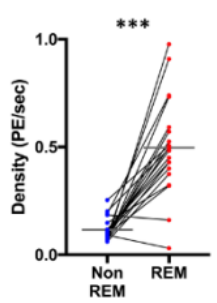

E

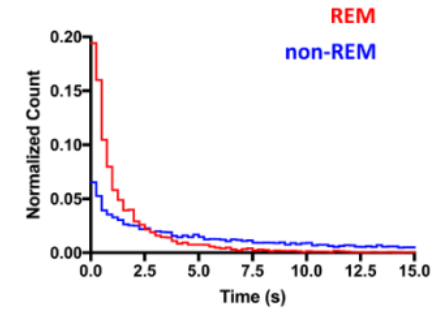

Phase locking to hippocampal theta phase

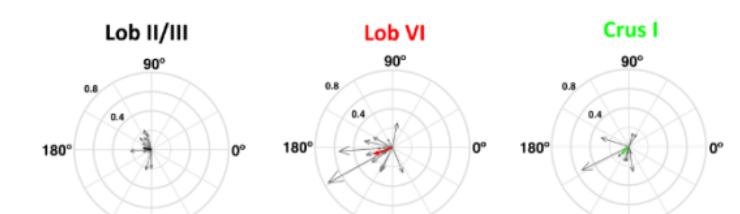

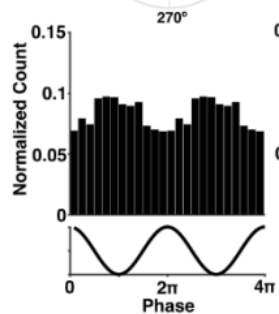

J
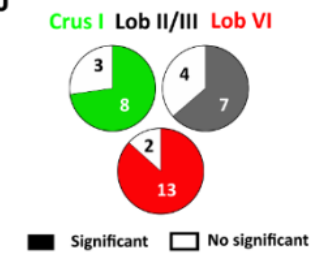

K

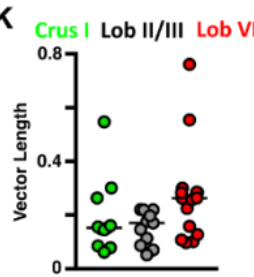

Figure 2. Phasic potentials (PSPs) occur at preferred phases of cerebellar delta and hippocampal

theta oscillations during REM. A, We observed phasic potentials in the cerebellar LFP recordings during REM (indicated by purple dots), which occurred preferentially during up-phase of the delta oscillations. Moreover, hippocampal theta frequency was transiently accelerated during the up-phase of the cerebellar delta oscillations. B, PSPs were found in all cerebellar recording sites and all mice. Averaged waveforms across animals (Lob II/III $n=11$; Lob VI $n=15$; Crus I $n=11$ ). C, Crosscorrelograms between the PSPs detected at a given cerebellar region and those recorded in the others. All cross-correlograms show a peak with $\leq 50 \mathrm{~ms}$ lag suggesting that most of the PSPs co-occurred at the three cerebellar regions near simultaneously. D, PSPs were observed during non-REM and REM sleep, however, the density of these events was significantly higher during REM (non-REM density = $0.1152 \pm 0.012 \mathrm{PSPs} / \mathrm{s}$; REM density $=0.4973 \pm 0.050 \mathrm{PSP} / \mathrm{s}$; paired t-test, $\mathrm{P}<0.0001)$. E, The distributions of the inter-event intervals (IEIs) were significantly different between non-REM and REM epochs (Two-sample Kolmogorov-Smirnov test, $\mathrm{P}<0.0001$ ). During REM, PSPs were preferentially 
concentrated as clusters (IEls $<1 \mathrm{~s}$ ). F, PSPs recorded at all cerebellar regions were phase-locked to the local delta oscillations during REM. In the top panels, the individual vectors of the phase locking for each mouse (grey arrows) and the average vector across all mice (colour coded; resultant vector angle: Crus I $=342.97^{\circ}, \mathrm{Lob} I \mathrm{I}=343.05^{\circ}, \mathrm{Lob} \mathrm{VI}=338.67^{\circ}$. Resultant vector length: Crus I $=0.34$, Lob $\mathrm{II}=0.28$, Lob VI $=0.41$ ). In the bottom panels, the normalized count of PSPs recorded at the different phases of delta oscillations in all mice for each cerebellar region (Lob II/III $=4004$ PSPs/11 mice, Rayleigh's test, $\mathrm{P}<0.001$; Lob VI $=3445$ PSPs $/ 15$ mice, Rayleigh's test, $\mathrm{P}<0.001$; Crus I $=3398$ PSPs $/ 11$ mice, Rayleigh's test, $P<0.001$ ). $G$, Fraction of mice with significant phase locking (significant phase locking was found in Crus I, 10/11 mice; Lob II/III, 11/11 mice; Lob VI 15/15 mice). H, The level of the phase locking did not differ across cerebellar regions (1-way ANOVA, $P=0.0932$ ). I-K, Same as for F-H but here calculating phase locking of PSPs detected in the cerebellum to ongoing hippocampal theta oscillations. I, Top row; Lob II/III Rayleigh's test, P < 0.001; Lob VI Rayleigh's test, P < 0.001; Crus I Rayleigh's test, $P<0.001$. J, Fraction of mice with significant phase locking of PSPS to hippocampal theta (significant phase locking was found in Crus I, 8/11 mice; Lob II/III, 7/11 mice; Lob VI 13/15 mice). $\mathbf{K}$, The level of the phase locking to hippocampal theta did not differ across cerebellar regions (KruskalWallis Test of vector lengths, $P=0.0717$ ).

\section{Intracerebellar cross-frequency modulation: cerebellar delta is a modulator of VHFOs during REM.}

The most prominent, ongoing LFP oscillations observed in the cerebellum during REM sleep were found in the delta $(<4 \mathrm{HZ})$ and VHFO $(\sim 250 \mathrm{~Hz})$ frequency ranges, respectively (see Figure 1$)$. Thus, to investigate any relationship between these two oscillators, we conducted cross-frequency spectral analysis (Figure 3). This revealed that during REM, the power of cerebellar VHFOs is significantly modulated by the phase of those in the delta range (Figure 3B, C). Interestingly, this modulation was significant only in vermal regions (Lob VI, II/III) and not in Crus I. This finding is consistent with the reduced presence of VHFOs during REM in this cerebellar region compared to the others recorded (Figure 1C, Supplemental Figure 1D), further indicating spatial heterogeneity in cerebellar processing during sleep.

Since PSPs were highly abundant during REM and also phase locked to cerebellar delta, we investigated whether they play a role in local cross-frequency modulation between delta phase and VHFO power. By comparing modulation in delta cycles with no PSPs, those containing isolated PSPs and those containing clusters of PSPs (Figure 3D), we found that PSP density did not significantly influence the degree of cross-frequency modulation observed in any of the recorded cerebellar regions (Figure 3D). 
233
A
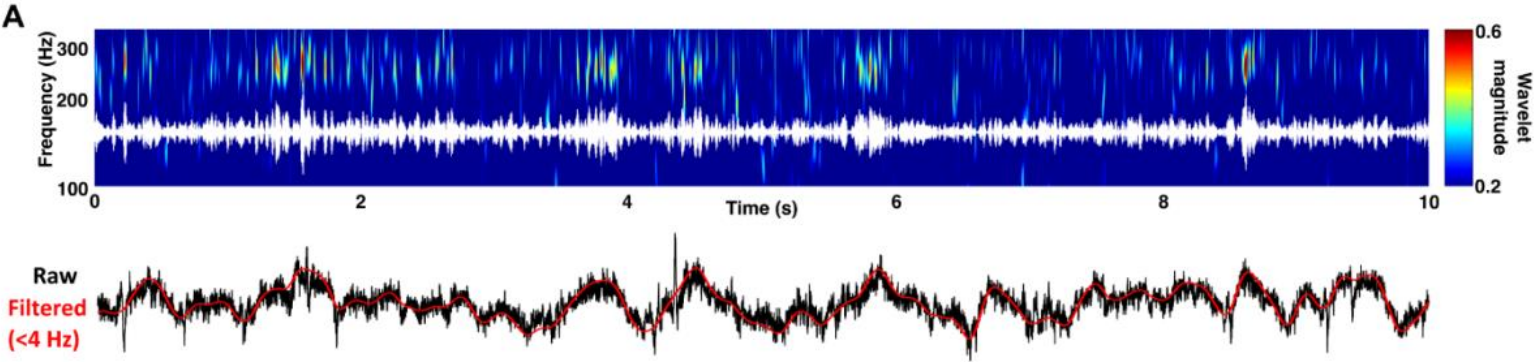

B
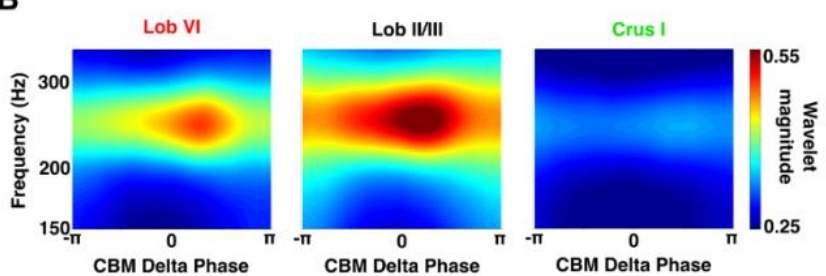

C

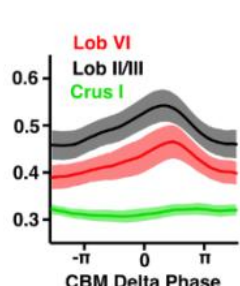

D

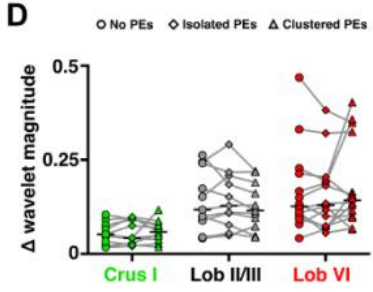

Figure 3. Modulation of VHFOs frequency oscillations by delta oscillations within the cerebellum during REM. A, Representative spectrogram (top; with VHFO frequency filtered trace overlaid in white) and raw cerebellar LFP recordings (bottom; with delta frequency filtered oscillations overlaid in red) showing the modulation of VHFOs by cerebellar delta oscillations during a REM epoch. The cerebellar LFP is dominated by both delta oscillations and VHFOs, which fluctuate dynamically within the $\sim 250 \mathrm{~Hz}$ and $<4 \mathrm{~Hz}$ ranges, respectively. The fluctuations in cerebellar VHFO power appear temporally aligned to the prominent delta oscillations in the cerebellar LFP. B, Average spectrograms aligned to the phase of the cerebellar delta oscillations $(<4 \mathrm{~Hz})$ during REM sleep. The power of cerebellar VHFOs appears greatest following the peak of cerebellar delta (zero phase radians) in vermal lobules (VI, II/III) but not Crus I. C, Average cerebellar VHFO power aligned to cerebellar delta oscillation phase (RM 2-Way ANOVA Cerebellar Region $x$ Delta Phase: Delta Phase $P<0.0001$, Cerebellar region $\mathrm{P}=0.0001$, Interaction $\mathrm{P}=\mathbf{0 . 0 0 0 2}$. Multiple comparisons of power across delta phase compared to trough value with FDR correction: Crus I: $p>0.05$, Lob II: $p<0.01$ from $-18.9^{\circ}-78.5^{\circ}(-$ $0.105 \pi-0.44 \pi$ ) Lob VI: $p<0.01$ from $\left.-3.72^{\circ}-93.97^{\circ}(-0.02 \pi-0.52 \pi)\right)$. D, Effect of PSP abundance during the cerebellar delta oscillations on the modulation of VHFOs during REM. We compared the maximal change in cerebellar VHFO power during cerebellar delta oscillations in which no PSPs, isolated PSPs or clusters of PSPs were detected. No significant differences were observed for the three cerebellar regions (2-Way ANOVA Cerebellar Region x Content of PSPs: Content of PSPS P $=0.8449$, Cerebellar Region $\mathrm{P}=0.003$, Interaction $\mathrm{P}=0.2601$, Multiple comparisons across cerebellar region effect with FDR correction: Crus I x Lob II, corrected $p=0.0101$, Crus I $\times$ Lob VI, corrected $p=0.0009$, Lob II $x$ Lob VI, corrected $p=0.1004$ ). In all mean plots, $n=$ Lob VI, $n=15$ mice; Crus I, $n=11$ mice; Lob II/III, $\mathrm{n}=11$ mice.

\section{Cerebellar delta and phasic sharp potentials modulate hippocampal theta oscillations during REM}

We next investigated if the prominent cerebellar delta oscillations and the high density of PSPS observed during REM was related to, or impacted upon, theta oscillations in the hippocampus, which are a well described physiological signature of REM sleep (Figure 4). During REM, hippocampal theta oscillations fluctuated within a frequency range of approximately $6-12 \mathrm{~Hz}$ and visual inspection of 
spectra and raw LFP traces revealed that these theta frequency fluctuations appeared to be temporally aligned with cerebellar delta oscillation cycles/PSP events (Figure 4A). Therefore, we next calculated hippocampal LFP power spectra triggered from the peak of cerebellar delta wave cycles. This analysis revealed that hippocampal theta frequency increased during the ascending phase of the cerebellar delta oscillation when compared with the values at the trough $(-\pi)$ (triggered from Crus I: maximal frequency of hippocampal theta $=7.97 \pm 0.095 \mathrm{~Hz}$ at $-18.74^{\circ}(-0.1 \pi)$; triggered from Lob II: maximal frequency of hippocampal theta $=7.94 \pm 0.095 \mathrm{~Hz}$ at $-3.72^{\circ}(-0.02 \pi)$; triggered from Lob VI: maximal frequency of hippocampal theta $=8.14 \pm 0.154 \mathrm{~Hz}$ at $-18.74^{\circ}(-0.1 \pi)$ Figure $\left.4 \mathrm{~B}, \mathrm{C}\right)$. oscillation cycles (Figure 2), we next investigated if the number of PSPs occurring during the cerebellar delta wave was associated with the level of hippocampal theta modulation. In contrast to intracerebellar delta phase modulation of VHFOs, by comparing cycles with no PSPs, those containing isolated PSPs and those containing clusters of PSPs (Figure 4D), we found that the acceleration of hippocampal theta was most prominent when clusters of cerebellar PSPs were present (PSP quantity $x$ cerebellar $<4 \mathrm{~Hz}$ phase repeated measures two-way ANOVA, quantity of PSP effect, $F(1,4)=43.94$, $p=0.0027$; interaction effect $F(47,188)=4.678, p$-value $<0.0001)$, suggesting that these phasic events are linked to the observed changes in hippocampal theta frequency.

A
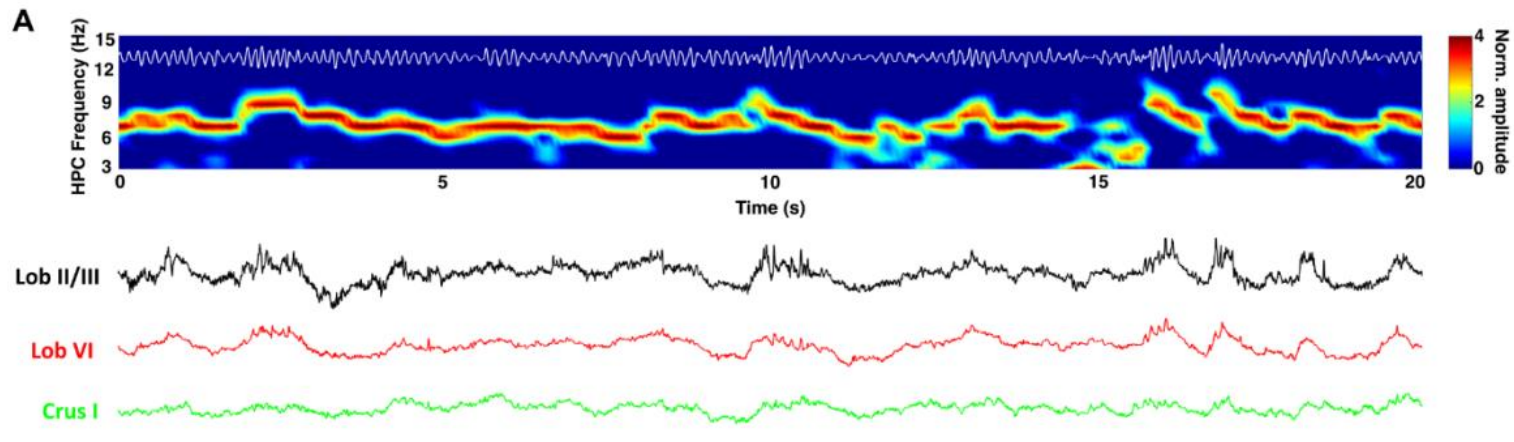

B

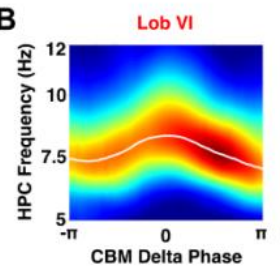

Lob IVIII

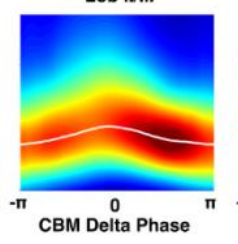

Crus।

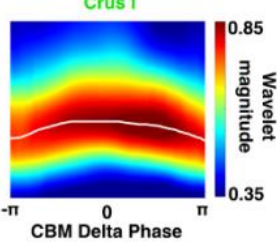

C

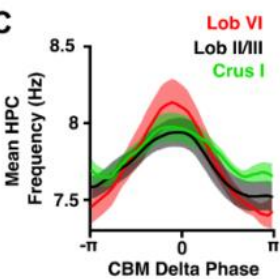

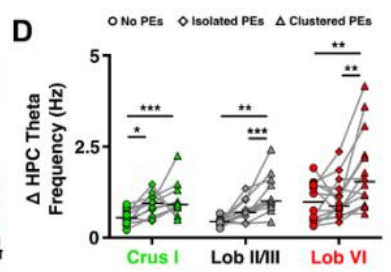

Figure 4. Modulation of hippocampal theta frequency by cerebellar delta oscillations during REM. recordings (bottom) showing the modulation of hippocampal theta frequency by cerebellar delta oscillations during a REM epoch. The hippocampal LFP is dominated by theta oscillations, which 
indicated by black horizontal bars above spectrogram). B, Example hippocampal spectrograms aligned to the phase of the cerebellar delta oscillations $(<4 \mathrm{~Hz})$ during REM sleep. The preferred hippocampal theta frequency (white line, overlaid) shows an acceleration coincident with the peak of the cerebellar delta oscillations (0 phase radians). C, Average preferred hippocampal theta frequency aligned to cerebellar delta oscillation phase (Lob VI, $\mathrm{n}=15$; Crus I, $\mathrm{n}=11$; Lob II/III, $\mathrm{n}=11$ ). Significant hippocampal theta frequency acceleration was found from the trough of delta to the peak of delta in all cerebellar regions. However, no differences in hippocampal theta frequency modulation were observed between the delta oscillations recorded across the three cerebellar locations (repeated measurements Two-way ANOVA phase $x$ cerebellar region, phase effect $p<0.0001$, region effect $p=$ 0.7953 , interaction effect $p=0.0295)$. $D$, Effect of PSP abundance during the cerebellar delta oscillations on the modulation of preferred hippocampal theta frequency during REM. We compared the maximal change in hippocampal theta frequency during cerebellar delta oscillations in which no PSPs, isolated PSPs or clusters of PSPs were detected. Significant differences were observed for the three cerebellar regions (Crus I, Friedman test with FDR correction, $p=0.0011$; Lob II/III, Friedman test with FDR correction, $p<0.0001$; Lob VI, Friedman test with FDR correction, $p=0.0015$ ) with a significant increase in the hippocampal theta modulation during delta waves when clusters of PSPS were detected compared with those with no PSPs or isolated PSPs.

\section{Hippocampal ripples drive LFP changes in the cerebellum}

During REM, dominant hippocampal theta oscillations are coordinated with prominent cerebellar LFP network activity (delta oscillations and PSPs). During non-REM sleep, however, hippocampal activity is characterized by the presence of prominent sharp-wave ripples (SWRs). Therefore, we next asked if cerebellar activity is coordinated or modulated by hippocampal SWRs during non-REM sleep. To address this question, we first detected SWRs (Figure 5A) and computed the cerebellar LFP averaged relative to SWR maximal amplitude (Figure 5B). Event related field potentials (ERPs) were clearly observed in all the recorded cerebellar regions. The SWR triggered responses were characterized by a prominent slow component that preceded the onset timing of SWR maximal amplitude followed by an ERP (occurring after SWR ripple maximal amplitude; Figure 5B). In order to compare the amplitude of the ERPs across cerebellar regions, we normalized them by subtracting the pre-SWR maximal baseline levels (Figure 5B inset). The onset-to-peak amplitude in normalized ERPs did not differ across cerebellar regions (Figure 5C, left panel). In contrast, latency to ERP peak was shortest in Lob VI (32 +- $7.89 \mathrm{~ms}$ ) compared to Crus I and to Lob II/III (Figure 5C, right panel).

We next asked whether the slow frequency component observed in the SWR-triggered responses resulted from the timing of SWRs at the peak of cerebellar slow oscillation cycles. To probe this question, we calculated phase locking of the detected hippocampal SWR relative to the phase of cerebellar delta oscillations ( $<4 \mathrm{~Hz}$, Figure 5D-F). We confirmed that hippocampal SWRs were phase locked to the delta rhythm observed in all three recorded cerebellar regions but that the strength of 
phase-locking was significantly greatest for Lob VI (Figure 5E, F). Given recent description of SWR propagation from hippocampus to neocortex (Nitzan et al., 2020), we also calculated SWR triggered spectral analysis of the cerebellar LFP (Supplemental Figure 4), which revealed no changes in the SWR frequency range $(\sim 150 \mathrm{~Hz})$ and thus suggests hippocampal SWRs do not propagate directly or indirectly to the cerebellum. Finally, we investigated potential interplay between PSPs recorded in the cerebellum and hippocampal SWRs. To do so, we computed the probability of detecting a SWR following the detection of a PSP. We found a consistent decrease in SWR probability $\sim 10 \mathrm{~ms}$ after the PSP peak in all cerebellar regions, which lasted for 1 sec (compared with a shuffled dataset; Figure $5 G)$. This reduction in SWR occurrence immediately following the PSPs suggests that the latter have an impact on local hippocampal activity.

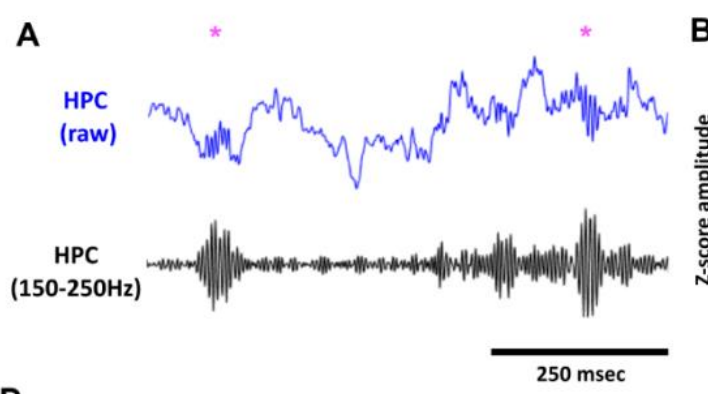

D

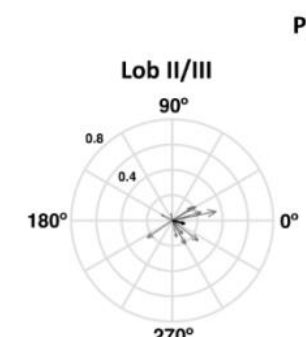

$270^{\circ}$

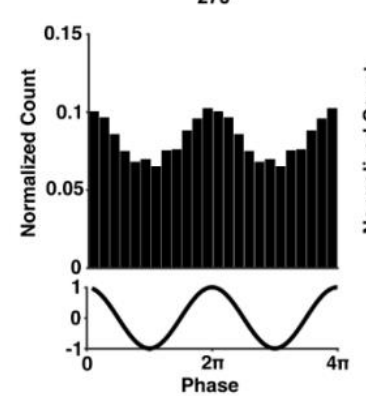

Phase locking to cerebellar delta phase

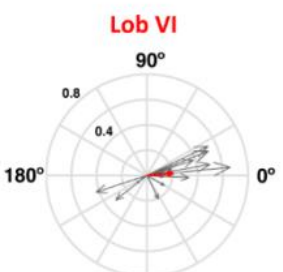

$270^{\circ}$

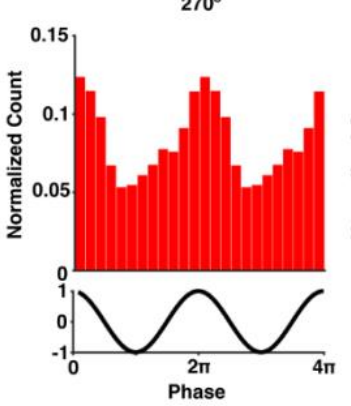

B

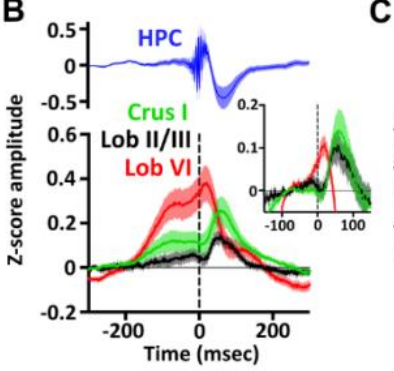

E

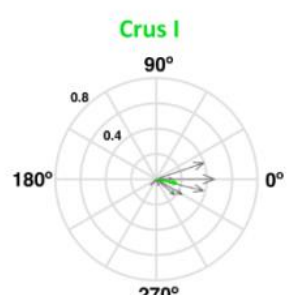

$270^{\circ}$

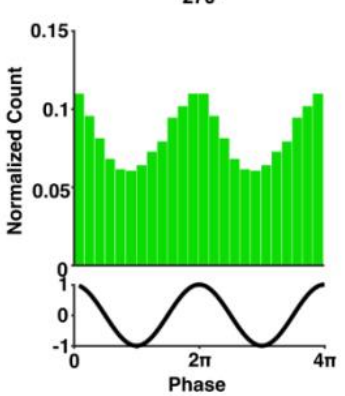

C
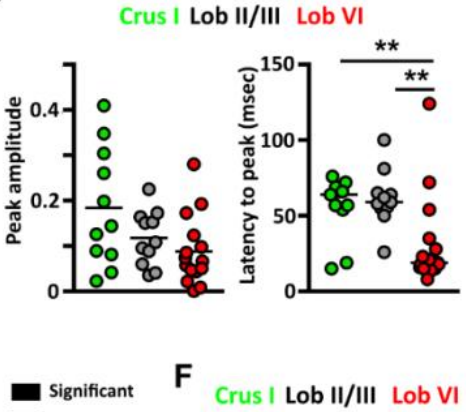
$F$ Crus I Lob II/III Lob VI

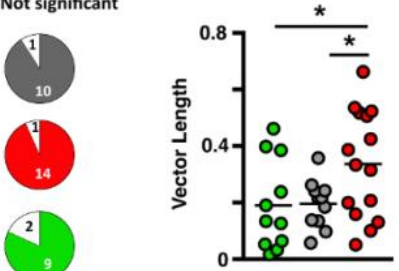

G

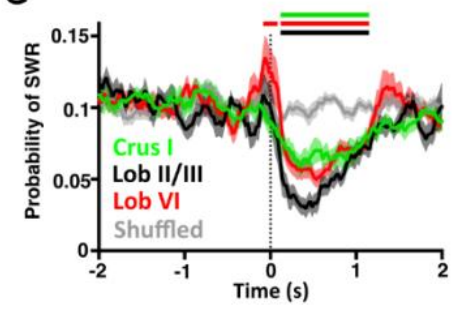

Figure 5. Hippocampal sharp-waves ripples (SWR) trigger evoked activity in the cerebellum.

A, Example of the raw (blue) and filtered (150-250 Hz, black) hippocampal LFP during non-REM sleep. SWR were identified (purple asterisks) at the maximal value in the filtered signal. B, Averaged SWRtriggered waveforms from the cerebellar recordings. Zero time represents the time of detected SWR peak in the filtered signal. Evoked field potentials were observed in all the cerebellar recordings. The averaged waveforms in Lob VI and Crus I revealed that the SWR occurred at the peak of cerebellar delta oscillations. Inset, shows the same data but baselined against values from -100 to $-50 \mathrm{msec}$, eliminating the slow frequency component and allowing clearer visualization of the evoked potentials. 
C, From the baseline normalised data, we quantified the ERP amplitude measured as the onset-topeak value and also the onset latency. No significant differences were observed for amplitude (Crus I, mean amplitude $=0.184+-0.04 ;$ Lob II, mean amplitude $=0.118+-0.02 ;$ Lob VI, mean amplitude $=$ $0.088+-0.02$; Kruskal-Wallis test $\mathrm{P}=0.0968$ ); however, Lob VI ERPs occurred at significantly shorter latencies compared to both Lob II/III and (Crus I: mean $=55.91+-6.14 \mathrm{~ms}$; Lob II/II: mean $=61.45+-$ $5.53 \mathrm{~ms}$; Lob VI: mean $=32+-7.89 \mathrm{~ms}$; Kruskal-Wallis test $\mathrm{P}=0.0053$; Multiple comparisons FDR corrected: Lob VI vs Crus I P= 0.0044 , Lob VI vs Lob II P= 0.0044 , Crus I vs Lob II P = 0.3034). D, Significant phase locking of SWRs to delta oscillations was found in all cerebellar regions during non-REM sleep. In the upper panels, the normalized count of PSPs recorded at the different phases of delta oscillations in all mice for each cerebellar region (Lob II/III = 14012 SWRs/11 mice, Rayleigh's test, $\mathrm{P}<0.001$; Lob $\mathrm{VI}=11915 \mathrm{SWRs} / 15$ mice, Rayleigh's test, $\mathrm{P}<0.001$; Crus I 15532 SWRs $/ 11$ mice, Rayleigh's test, $\mathrm{P}<$ 0.001). In the lower panels, the individual vectors of the phase locking for each mouse (grey arrows) and the average vector across all mice (colour coded; resultant vector angle: Crus I $=348.27^{\circ}$, Lob II = $345.4^{\circ}, \mathrm{Lob} \mathrm{VI}=4.26^{\circ}$. Resultant vector length: Crus I $=0.16$, Lob II $=0.1$, Lob VI $=0.21$ ). E, Fraction of mice with significant SWR-to-cerebellar delta phase locking (significant phase locking was found in Crus I, 9/11 mice; Lob II/III, 10/11 mice; Lob VI 14/15 mice). F, The level of SWR phase locking was higher with Lob VI compared to Crus I or Lob II/III (1-way ANOVA, $\mathrm{P}=0.0324$; multiple comparisons FDR corrected, Lob VI-Crus I P = 0.0149, Lob VI-Lob II/III P = 0.0149, Crus I-Lob II/III P=0.3317). G, Probability of SWRS relative to PSP onset. The number of SWRs are reduced following PSP occurrence (at time zero; cf. Tsunematsu et al., 2020; 2-way ANOVA, region vs time, time effect $P<0.0001$, region effect $P<0.0001$, interaction effect $P<0.0001$. Multiple comparisons between cerebellar regions and shuffled data)._For all mean plots, Crus $1, n=11$ mice; Lob II/III, $n=11$ mice; Lob VI, $n=15$ mice.

\section{Discussion}

Despite the extensive literature on sleep-related neurophysiological processes in the hippocampus (for review, see Klinzing et al., 2019) and recent advances in understanding of sleep-related processes in the cerebellum (Canto et al., 2017; Xu et al., 2020; Zhang et al., 2020), no report exists on potential functional or physiological interplay between these regions during sleep. Therefore, to bridge this knowledge gap, we investigated cerebello-hippocampal interactions as well as intrinsic cerebellar LFP dynamics across sleep states in mice.

We first profiled sleep-related activity within three distinct regions of the cerebellar cortex: Lob VI, Lob II/III and Crus I. We found modulation of cerebellar LFP activity across sleep states, both locally, in spatially segregated cerebellar lobules, and globally, in a coordinated manner across the cerebellar cortex. Overall, during REM and non-REM sleep, prominent delta $(<4 \mathrm{~Hz})$ and VHFOs $(\sim 250 \mathrm{~Hz})$ dominate the spectral profile in all three regions. The presence of VHFOs has been described previously in vermal lobules V/Vla of anaesthetised and awake, head-fixed rats, and attributed to 
activity in recurrent Purkinje cell collaterals (de Solages et al., 2008). In contrast, previous studies in freely moving mice have predominantly described high frequency oscillations in the cerebellar cortex ( $150 \mathrm{~Hz}$ : Cheron et al., 2005, 2004; Servais et al., 2005), which is consistent with our observations during wake. Thus, given the high presence of VHFOs in head-fixed or sleeping animals they may be related to the absence of voluntary movements. Our findings additionally extend the work of de Solages et al. (2008) by showing for the first time that VHFOs are concurrently present across multiple cerebellar cortical regions, highly prevalent during sleep (above wake levels) and, furthermore, that they are temporally coordinated by local cerebellar delta rhythms during REM sleep.

During non-REM sleep, our description of prominent delta oscillations in the cerebellar cortex is in keeping with previous work showing that neocortical slow oscillations can entrain those in the cerebellar cortex (Roš et al., 2009a; Rowland et al., 2010; Xu et al., 2020). In contrast, REM sleep is traditionally associated with theta $(\sim 6-12 \mathrm{~Hz})$ frequency, LFP oscillations. Our recordings from the cerebellum revealed no discernible increase in theta power during REM. Surprisingly, however, we did observe highly synchronous, large delta $(<4 \mathrm{~Hz})$ oscillations in all three recorded cerebellar regions during REM. This may be a local circuity peculiarity; however, recent evidence rodent and human studies suggest that localized delta oscillations should be considered an integral component of REM sleep (Bernardi et al., 2019; Funk et al., 2016; Siclari and Tononi, 2017).

Next, we sought evidence for putative functional coupling, as measured by LFP-LFP coherence, between the hippocampus and cerebellum across sleep states. Of particular note, we found that hippocampus-Lob VI coherence was significantly and dynamically modulated across sleep states.

400 Specifically, during non-REM, hippocampus-Lob VI delta coherence was significantly higher than 401 during wake/REM whilst during REM, hippocampus-Lob VI theta coherence was significantly higher 402 than during non-REM and, surprisingly, was also significantly modulated to above wake levels. The 403 comparatively heightened, frequency-specific, offline coupling between hippocampus and Lob VI during non-REM illustrates the potential for both sleep stage and regionally specific interactions between the two brain structures in addition to those we previously described during active movement in the homecage (Watson et al., 2019). Indeed, this interaction may be supported by 
411 In addition to the ongoing LFP oscillations observed in the cerebello-hippocampal network during 412 sleep, we also detected high amplitude PSPs in the cerebellar cortical recordings that were particularly 413 abundant during REM sleep. Previous studies have described similar phasic events in the cerebellum 414 and attributed them to propagation of PGO waves (Farber et al., 1980; Velluti et al., 1985). In keeping 415 with our observations, PGO waves are found to be highly concentrated in REM epochs across multiple 416 brain regions (Harlay et al., 1974; Marks et al., 1980; Pellet and Harley, 1977; Tsunematsu et al., 2020). 417 Furthermore, PGO-waves are known to phase-lock to hippocampal theta rhythms during REM and 418 modulate its frequency (increasing the preferred theta frequency; Karashima et al., 2007, 2002). We 419 confirmed that this was also the case with phase-locking of cerebellar PSPs to the hippocampal LFP 420 theta oscillation. Additionally, we also show that cerebellar PSPs are significantly phase-locked to the 421 cerebellar delta oscillation cycle and cross-frequency analysis revealed that cerebellar delta 422 modulation of hippocampal theta oscillations occurs preferentially when PSP content is high. Thus, 423 overall, the most parsimonious explanation is that the PSPs we recorded in the cerebellum are 424 propagated PGO waves. During REM, PGOs have been shown to play a crucial role in coordinating long 425 range network dynamics underlying sleep-dependent cognitive processes required for establishment 426 of fear memory (Datta, 2000; Datta et al., 1998; Datta and O'Malley, 2013). It is tempting to speculate 427 that the PSPs observed in the cerebellum may play a similar role by coordinating interactions with the 428 hippocampus and that this could subserve sleep-dependent memory formation of the explored 429 environment.

430 Sharp wave ripples link hippocampus and cerebellum during non-REM sleep

431 Sharp wave ripples (SWRs) are a prominent physiological feature of hippocampal activity during non432 REM sleep. SWRs are fast oscillations during which both CA3 and CA1 pyramidal cells fire 433 synchronously (Buzsáki, 1986; Csicsvari et al., 2000). Their occurrence is coordinated with cortical 434 spindles, which are themselves synchronized with cortical slow-delta oscillations (Siapas and Wilson, 435 1998) and this tripartite interaction is important for memory formation (Girardeau et al., 2009; 436 Latchoumane et al., 2017; Maingret et al., 2016). Consequently, we next examined the relationship 437 between hippocampal SWRs and cerebellar LFP oscillations. SWR-triggered cerebellar LFP waveform 438 averages in combination with phase-locking analysis revealed two main findings. Firstly, ERPs are 439 present in the cerebellar cortical LFP at short latency (particularly in Lob VI) following hippocampal 440 SWR onset. Previously, hippocampal SWRs have been shown to drive evoked LFP and single-unit 441 changes in the cingulate cortex of a similar latency to those triggered by direct hippocampal electrical 442 stimulation; as such SWR activity may be considered indicative of efferent flow from the hippocampal 
formation (Wang and Ikemoto, 2016). Indeed, we found that SWRs triggered cerebellar ERPs with a short onset latency of $\sim 9.17 \mathrm{~ms}$, which is in agreement with previous reports in anaesthetised rats (Saint-Cyr and Woodward, 1980; Saint-Cyr and Woodward, 1980b) in which direct electrical stimulation of the hippocampal fornix elicited both short latency mossy fibre (5-10 ms, routed via the pontine nuclei) and longer latency, climbing fibre (10-20 ms, routed via the inferior olive) responses within the cerebellar cortex. Also consistent with our results, topographical mapping of cerebellar responses following direct hippocampal electrical stimulation revealed evoked activity mainly in the Lob VI region of both cats (Newman and Reza, 1979) and rats (Saint-Cyr and Woodward, 1980b). Given the short latency of evoked SWR ERPs, particularly in Lob VI, it seems likely that SWR mediated hippocampal input to the cerebellum during non-REM sleep could be routed via the pontine nuclei. This hypothesis is further strengthened by evidence of a direct hippocampal projection to the pons

454 (Schmahmann and Pandya, 1997).

Our second main finding was that SWRs are significantly phase locked to the up-state of cerebellar delta oscillations in striking similarity to the well described locking of SWRs to neocortical delta, which is thought to facilitate transfer of memory information from subcortical to cortical loci (Maingret et al., 2016). As cerebellar Purkinje cells are known to toggle between depolarizing 'up-states' and hyperpolarizing 'down-states' (see Engbers et al., 2013 for review) it may be considered that, during non-REM sleep, cerebellar delta oscillations may facilitate the transfer of hippocampal information via phase-locking of SWRs to periods of high Purkinje cell excitability thus favouring plasticity mechanisms associated with memory formation. Consistent with our finding that hippocampus - Lob VI delta coherence during non-REM sleep is higher compared to other cerebellar regions, SWRs were significantly more phase locked to Lob VI delta compared to Crus I or Lob II/III, further supporting the potential for regionally and sleep stage specific hippocampal-cerebellar interactions. Another physiological explanation of SWR links to cerebellar delta oscillations may be provided by the fact that SWRs are known to nest to the upstate of neocortical slow-delta oscillations. In turn, these neocortical oscillations have been shown to entrain cerebellar activity in both anaesthetised rats (Roš et al., 2009b; Rowland et al., 2010; Schwarz, 2010) and naturally sleeping monkeys (Xu et al., 2020). Thus, SWR phase-locking to cerebellar delta oscillations may be mediated via upstream SWR-to-neocortical delta nesting.

Taken together, the SWR-triggered evoked LFP activity recorded in the cerebellar cortex and their coordination with cerebellar delta oscillations illustrate the existence of robust physiological links and candidate mechanisms that could subserve hippocampal-cerebellar (particularly Lob VI) interactions during non-REM sleep. 
476 In summary, our findings illustrate the presence of multiple physiological events in the cerebello-

477 hippocampal network during sleep (Supplemental Figure 5), centred around prominent delta

478 oscillations. In particular, we have highlighted lobule specific hippocampus-to-cerebellum directed

479 interaction during non-REM sleep mediated via SWR and delta oscillations. During REM, we identified

480 prominent cerebellar delta oscillations and associated PSPs, which modulate both local cerebellar

481 VHFO and distant hippocampal theta oscillations. Thus, it appears that delta oscillations play a key

482 role in temporal coordination both within and between these regions. Similar sleep-stage specific,

483 transient physiological events have been shown to be important for memory formation across other

484 brain circuits, and by analogy it may be hypothesised that the events described in the hippocampal-

485 cerebellar network serve a similar role in affording the two regions the ability to preferentially interact

486 during windows of enhanced synaptic plasticity.

487 As the cerebellum is thought to compute forward models that predict sensory consequences of actions 488 adapted to a particular context, we speculate that the aforementioned mechanisms may allow offline 489 updating and refinement of such models across the cerebello-hippocampal network during sleep. 490 These refinements may be important during online navigation behaviours in which animals must 491 predict the spatial consequences of their movements. 
Material and Methods:

496

497

498

499

500

501

502

503

504

505

506

507

508

509

510

511

512

513

514

515

516

517

518

519

520

521

522

523

524

525

526

Mice

20 adult male C57BL6-J mice were used for this study (Janvier, France). Mice were housed individually under a $12 \mathrm{hr}$ light/12 hr dark cycle (light cycle beginning at $8 \mathrm{am}$ ) and received food and water ad libitum. All behavioural experiments were performed in accordance with the official European guidelines for the care and use of laboratory animals (86/609/EEC) and in accordance with the Policies of the French Committee of Ethics (Decrees no 87-848 and no 2001-424). The animal housing facility of the laboratory where experiments were made is fully accredited by the French Direction of Veterinary Services (B-75-05-24, 18 May 2010). The protocol was approved by the Committee on the Ethics of Animal Experiments (APAFIS\#4315-2016042708195884v1). Data obtained from the mice used in this study have been published in Watson et al., (2019).

\section{Surgery}

Surgical and implant procedures have been already described in Watson et al., (2019). Briefly, surgical implantation was performed under constant isoflurane anaesthesia (1.5\%) combined with oxygen (1.5 $\mathrm{L} / \mathrm{min}$ ). Animals were placed in a stereotaxic frame device (David Kopf Instruments, USA) and an incision was performed in order to expose the scalp. Coordinates for implantation were calculated from bregma following the references given in Franklin and Paxinos, 2007. We targeted bilateral hippocampi (AP -2.2 mm, ML $\pm 2.0 \mathrm{~mm}$, DV $1.0 \mathrm{~mm}$ ), cerebellar lobules II/III (AP -5.52 mm, ML $0 \mathrm{~mm}$, DV $1.8 \mathrm{~mm}$ ), cerebellar lobule VI (AP $-6.72 \mathrm{~mm}, \mathrm{ML} 0 \mathrm{~mm}$, DV $0.1 \mathrm{~mm}$ ) and left cerebellar Crus I (AP $6.24 \mathrm{~mm}, \mathrm{ML} 2.5 \mathrm{~mm}$, DV $0.1 \mathrm{~mm}$ ). Small craniotomies were performed over the target regions using a drill and the dura was carefully removed with a needle. Two wires of $140 \mu \mathrm{m}$ diameter teflon coated stainless-steel (A-M system, USA) were twisted together to create bipolar LFP recording electrodes (interpolar distance $\sim 0.5 \mathrm{~mm}$ ) and were implanted in the brain. Pairs of flexible stainless-steel wires were also sutured to the neck muscles to obtain EMG recordings (Cooner wire, USA). 14 mice were also implanted with bipolar stimulation electrodes (same as the LFP electrodes but with interpolar distance of $\sim 140 \mu \mathrm{m}$ ) in the left medial forebrain bundle (AP $-1.4 \mathrm{~mm}, \mathrm{ML} 1.2 \mathrm{~mm}$, DV $4.8 \mathrm{~mm}$ ) to serve as a reward signal in a set of experiments already published (Watson et al., 2019). All electrodes were attached to an electrode interface board (EIB-18, Neuralynx, USA) and the assembly was fixed to the skull using a combination of UV activated cement (SpeedCem, Henry Shein, UK), SuperBond (SunMedical, Japan) and dental cement (Simplex Rapid, Kemdent, UK). Four miniature screws (Antrin, USA) were also attached to the skull for additional support and to serve as recording ground. Animals were given a minimum of 5 days post-surgery recovery time before experiments commenced. 
528 The EMG and LFP recordings were obtained via a unity-gain headstage preamplifier (HS-18, Neuralynx, 529 USA) and a Digital Lynx SX system and Cheetah software (Neuralynx, USA). Signals were bandpass530 filtered between 0.1 and $600 \mathrm{~Hz}$ and sampled at $1 \mathrm{kHz}$. Mouse position was tracked at $30 \mathrm{~Hz}$ using 531 video tracker software and infra-red LEDs attached to the headstage (Neuralynx, USA).

532 Histology

533 After completion of the experiments, mice were deeply anaesthetized with ketamine/xylazine 534 solution $(150 \mathrm{mg} / \mathrm{Kg})$ and electrolytic lesions were created by passing a positive current through the 535 electrodes $(30 \mu \mathrm{A}, 10 \mathrm{~s})$. The animals were then perfused transcardially with saline $(0.9 \%)$ followed 536 by paraformaldehyde (4\%). Once perfused, the electrode assembly was carefully removed, the brain 537 extracted and post-fixed in paraformaldehyde (4\%) for $24 \mathrm{hr}$ and then embedded in agarose (3\%). 50 $538 \mu \mathrm{m}$ sagittal and coronal sections for the cerebellum and the hippocampus, respectively, were made 539 using a vibratome. The sections were mounted on gelatinized slides and stained with cresyl violet. The 540 electrolytic lesions were then identified in order to reconstruct the recording locations using standard 541 maps with reference to a stereotaxic atlas (Franklin and Paxinos, 2007). The anatomical location of the 542 electrodes used in this study were verified in Watson et al., (2019).

\section{$543 \quad$ Behaviour and sleep scoring}

544 The animals were recorded during the day (between $10 \mathrm{am}$ and $6 \mathrm{pm}$ ) in their home-cages $(30 \mathrm{~cm} \mathrm{x}$ $54510 \mathrm{~cm} \times 10 \mathrm{~cm}$ ), with the lid removed and the lights off for periods up to $4 \mathrm{hr}$. Recordings were made 546 prior to training in a linear track task (described in Watson et al., 2019). The homecage environment 547 was familiar to the mice as they had been housed in it since completion of implantation surgery. 548 Animals exhibited different behavioural states that were scored off-line and included in four 549 categories: active wakefulness, resting, non-REM sleep and REM sleep. The scoring was semiautomatically performed using multi-parameter thresholds based upon instantaneous speed, neck EMG (rectified, smoothed with $1 \mathrm{~s}$ window and z-scored) and theta/delta ratio in the hippocampal LFP (z-scored and calculated in $100 \mathrm{~ms}$ bins). Thus, epochs of more than $4 \mathrm{~s}$ with instantaneous speed above $3 \mathrm{~cm} / \mathrm{s}$ were considered as active wakefulness, periods of less than $30 \mathrm{~s}$ where the speed stayed below $3 \mathrm{~cm} / \mathrm{s}$ and were surrounded by active wakefulness epochs were considered as rest. Similarly, when the EMG recording was optimal, a manually selected threshold was also used to discriminate between rest and sleep epochs. We finally used manual thresholding of the theta/delta ratio to separate non-REM and REM sleep epochs. Only epochs of more than four seconds were selected for further analysis. 
All the data processing was performed using custom-made MATLAB scripts (Mathworks, USA). Raw signals were pre-processed by applying a notch filter to remove electrical line noise (filter centred to $50 \mathrm{~Hz}$ ). Voltages were z-scored to reduce overall differences in amplitude in the signal recorded from different electrodes.

\section{Offline detection of ripples, slow oscillations and phasic events}

565 Discrete sharp-waves ripples (SWRs) were detected using criteria employed elsewhere (Maingret et

566 al., 2016). The raw signal was then filtered using a second order zero-phase bandpass filter between

567 150-250 Hz, squared, smoothed (using $8 \mathrm{~ms}$ running average) and z-scored. The SWRs were defined

568 as events in which the transformed signal remained above $2 \mathrm{z}$-scores for 30 to $100 \mathrm{~ms}$ with a peak

569 above 5 z-scores.

570 Slow oscillations in the cerebellum were divided into individual cycles which were identified as follows:

571 first the raw signal was filtered using a second order zero-phase bandpass filter between $0-1-4 \mathrm{~Hz}$. The 572 instantaneous phase was obtained by applying a Hilbert transform to the filtered signal. Each cycle 573 was defined by the epoch between two negative peaks on the cosine of the instantaneous phase. Only 574 cycles which lasted more than $1 \mathrm{~s}$ and presented peak amplitudes higher than $4 \mathrm{z}$-scores and trough 575 amplitudes no lower than $2 \mathrm{z}$-scores were further analysed.

576 For the detection of PSPs (putative p-waves) in the cerebellum, we first filtered the signal using a 577 second order zero-phase bandpass filter between $5-80 \mathrm{~Hz}$, squared it, and then calculated the z-score 578 across all obtained sleep epochs. A double threshold strategy was then applied, first to the 579 transformed signal so that epochs separated by at least $75 \mathrm{~ms}$ with values above $1 \mathrm{z}$-score and a peak in the filtered data above $4 \mathrm{z}$-scores were considered as events.

\section{$581 \quad$ Spectral analysis}

582 All the spectral analyses were performed employing freely available signal processing toolboxes. For 583 computing the overall spectral power and spectral coherence across sleep-states, a multi-taper 584 Fourier transform (Chronux toolbox) was computed with $4 \mathrm{~s}$ sliding windows in $1 \mathrm{~s}$ steps and using 4 tapers. The mean power spectrum and coherence was obtained for each behavioural state by averaging the spectrogram and coherogram from the identified epochs. To reduce the impact of the different data durations for each state, we split the epochs to match with the minimal data duration and then we averaged them. For computing the triggered spectrogram and coherograms we used the 
continuous wavelet transform and wavelet coherence (wavelet toolbox, MATLAB). We used analytic Morse wavelets with 8 octaves and 48 voices per octave.

$591 \quad$ Phase locking of phasic events and slow-wave triggered power spectra and coherograms

592 Cerebellar and hippocampal signals were filtered using a second order zero-phase bandpass filter 593 between $0.1-4 \mathrm{~Hz}$ and $6-12 \mathrm{~Hz}$, respectively. The instantaneous phase of the cerebellar infra-slow 594 oscillations and the hippocampal theta oscillations were obtained by applying a Hilbert transform to 595 the filtered signals. The phase locking of the detected cerebellar phasic events to these rhythms was tested using circular statistics (Matlab) and significance was determined as Raleigh test $p$-value $<0.05$.

To compute the cerebellar slow-wave triggered spectrogram and coherogram, for each cerebellar slow-wave, the obtained time-frequency series was matched with the corresponding instantaneous phase of the cerebellar delta oscillations $(0.1-4 \mathrm{~Hz})$ and then averaged by phase bins of $7.35^{\circ}$.

\section{Cross-correlation between PSPs and SWRs}

601 The timing of the SWRs was calculated relative to the time of the peak of the detected PSPs. After 602 binning at 25 ms resolution, the probability density function (count normalized by the total number 603 of elements multiplied by the bin width) was obtained and smoothed (using a 250 ms running 604 average). Statistical significance of the obtained cross-correlation was performed by artificially 605 generating PSP trains of similar density as observed in the actual data, drawn at random from a Poisson 606 distribution, and recomputing the probability density function.

\section{$607 \quad$ Statistical analysis}

608 Statistical analyses were conducted using MATLAB Statistical Toolbox and Prism (Graphpad, USA). 609 Normality was assessed using a Shapiro-Wilk test. Parametric and non-parametric tests were then 610 used accordingly. Paired analyses were employed when possible.

\section{Author Contributions}

613 Arturo Torres-Herraez, Software, Formal analysis, Validation, Investigation, Visualization,

614 Methodology, Writing- original draft; Thomas Charles Watson, Conceptualization, Supervision, Formal 615 analysis, Validation, Visualization, Investigation, Methodology, Writing- original draft, review and 616 editing; Laure Rondi-Reig, Conceptualization, Resources, Supervision, Funding acquisition, 617 Methodology, Writing-critical revision, review and editing, Project administration. 
bioRxiv preprint doi: https://doi.org/10.1101/2021.05.04.442571; this version posted May 4, 2021. The copyright holder for this preprint (which was not certified by peer review) is the author/funder, who has granted bioRxiv a license to display the preprint in perpetuity. It is made available under aCC-BY-NC-ND 4.0 International license.

\section{Competing interest}

620 The authors declare that no competing interests exist.

621

622

Acknowledgments

623 This work was supported by the Fondation pour la Recherche Médicale DEQ20160334907-France, the

624 National Agency for Research ANR-17-CE16-0019-SynPredict, CNRS, Inserm and Sorbonne University

625 (LRR). We thank all members of the CEZAME team for helpful discussions of the experiments and 626 manuscript. We gratefully acknowledge the IBPS animal facility staff for their support.

627

628 
A

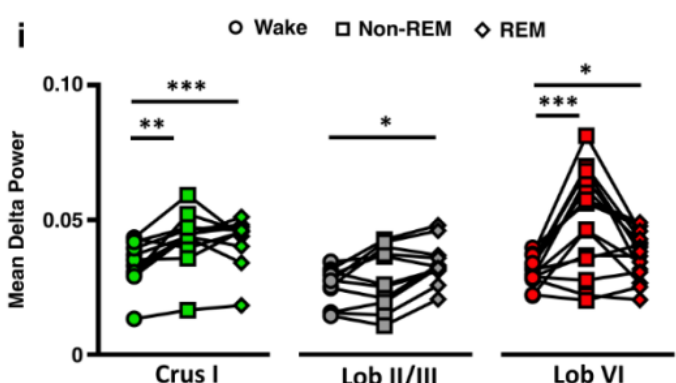

$B_{\mathbf{i}}$

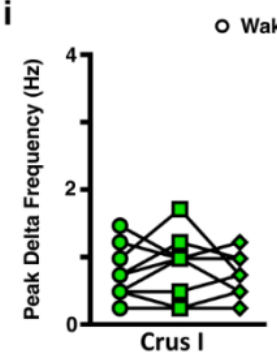

Wake $\square$ Non-REM $\diamond$ REM

C

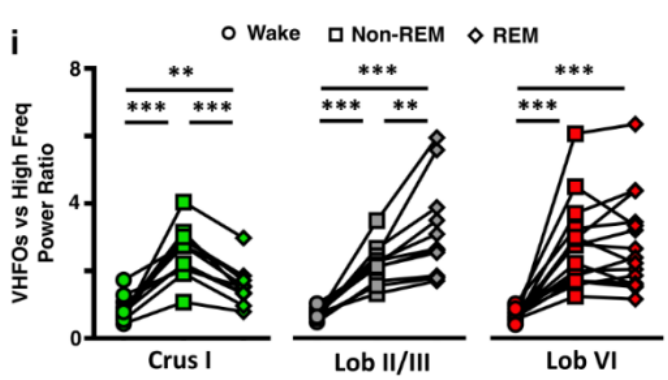

$\mathbf{D}_{\mathbf{i}}$

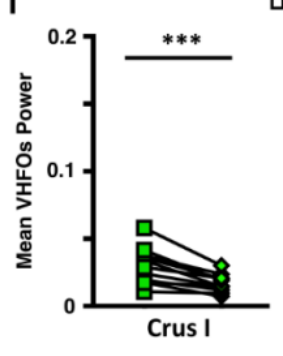

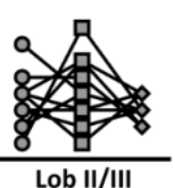

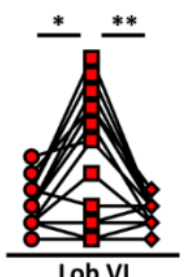

Lob VI

Supplemental Figure 1. LFP power in delta (0.1-4 Hz Hz) and VHFO (240-280 Hz) frequency bands during wake and sleep. $\mathbf{A}, \mathbf{i}$, The power of delta oscillations varied between wake and the different sleep states within the three cerebellar regions (Crus I, $n=11$, Friedman test, $p=0.0004$, multiple comparisons with FDR correction: awake vs non-REM, $p=0.0015$, awake vs REM, $p=0.0007$, non-REM vs REM, $p=0.2344$; Lob II/III, $n=11$, Friedman test, $p=0.0273$, multiple comparisons with FDR correction: awake vs non-REM, $p=0.3657$, awake vs REM, $p=0.0221$, non-REM vs REM, $p=0.0578$; Lob VI, $\mathrm{n}=15$, Friedman test, $\mathrm{p}=0.0023$, multiple comparisons with FDR correction: awake vs nonREM, $p=0.0011$, awake vs REM, $p=0.0468$, non-REM vs REM, $p=0.1009$ ). ii, Similarly, differences between cerebellar regions were observed both during wake (Kruskal-Wallis test, $p=0.0133$, multiple comparisons with FDR correction: Crus I vs Lob II/III, $p=0.0075$, Crus I vs Lob VI, $p=0.1473$, Lob II/III vs Lob VI, $p=0.0633$ ) and non-REM sleep (Kruskal-Wallis test, $p=0.0040$, multiple comparisons with

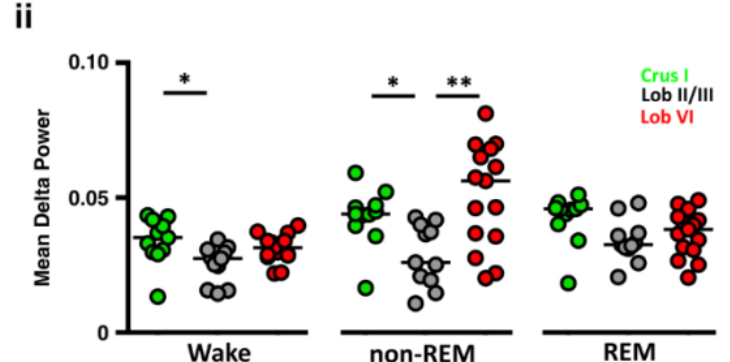

ii
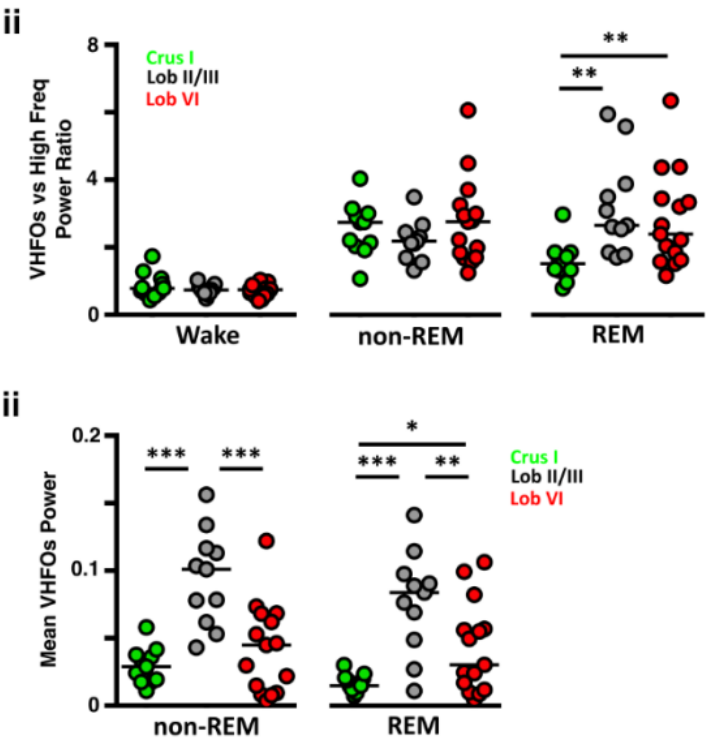
0.0012), but not during REM (Kruskal-Wallis test, $p=0.0540$ ). B, $\mathbf{i}$, The peak delta band frequency differed across sleep states only in Lob VI (Crus I, Friedman test, $p=0.1322$; Lob II/III, Friedman test, $p=0.2233$; Lob VI, Friedman test, $p=0.009$, multiple comparisons with FDR correction: awake vs nonREM, $p=0.0107$, awake vs REM, $p=0.1955$, non-REM vs REM, $p=0.0039$ ). ii, Differences in the peak delta frequency band across cerebellar regions were also restricted to non-REM epochs (wake, Kruskal Wallis test, $p=0.7732$, non-REM, Kruskal Wallis test, $p=0.0364$, multiple comparisons with FDR correction: Crus I vs Lob II/III, $p=0.9095$, Crus I vs Lob VI, $p=0.0596$, Lob II/III vs Lob VI, $p=0.0596$ ). $\mathrm{C}, \mathrm{i}$, During wake, the power of VHFOs was lower compared to high frequency oscillations in all regions, as indicated by the mean values below 1. In contrast, during sleep, across all recorded cerebellar regions, the ratio of VFHO to high frequency oscillation power increased significantly compared to wake (shifted to values $>1$ ), and also differed between sleep states (Crus I, repeated measures ANOVA, $p<0.0001$, multiple comparisons with FDR correction: awake vs non-REM, $p<0.0001$, awake vs REM, $p=0.0046$, non-REM vs REM, $p<0.0001$; Lob II/III, repeated measures ANOVA, $p=0.0002$, multiple comparisons with FDR correction: wake vs non-REM, $p<0.0001$, wake vs REM, $p=0.0003$, non-REM vs REM, $p=0.0071$; Lob VI, repeated measures ANOVA, $p<0.0001$, multiple comparisons with FDR correction: wake vs non-REM, $p<0.0001$, wake vs REM, $p<0.0001$, non-REM vs REM, $p=0.7226$ ). ii, Significant differences across cerebellar regions were restricted to REM epochs when Crus I shown significantly smaller ratios than the other two regions (wake, one-way ANOVA, $p=0.2252$, non-REM, one-way ANOVA, $p=0.3705$, REM, one-way ANOVA, $p=0.0098$, multiple comparisons with FDR correction, Crus I vs Lob II/III, $p=0.0042$, Crus I vs Lob VI, $p=0.0083$, Lob II/III vs Lob VI, $p=0.1538$ ). $D, i$, VHFO power was significantly reduced during REM sleep compared with non-REM in Crus I (Paired t-test, $p=0.0005$ ) and Lob II/III (Paired t-test, $p=0.0067$ ) but remained unchanged in Lob VI (Paired t-test, $p=0.9137$ ). ii, VHFOs power was significantly higher in Lob II during both non-REM (one-way ANOVA, $p<0.0001$, multiple comparisons with FDR correction, Crus I vs Lob II/III, $p<0.0001$, Crus I vs Lob VI, $p=0.1020$, Lob II/III vs Lob VI, $p<0.0001$ ) and REM sleep (one-way ANOVA, $p=0.0002$, multiple comparisons with FDR correction, Crus I vs Lob II/III, $p=0.0001$, Crus I vs Lob VI, $p=0.0393$, Lob II/III vs Lob VI, $p=0.0092$ ).
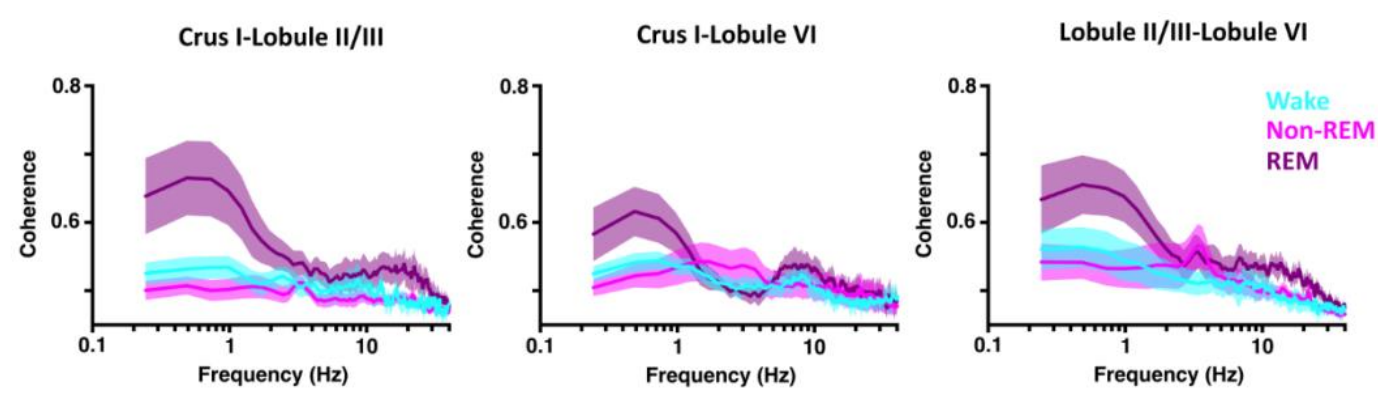

672 Supplemental Figure 2. Intra-cerebellar delta coherence is highest during REM sleep. Delta band (< $6734 \mathrm{~Hz}$ ) coherence was elevated during REM sleep compared with both awake and non-REM in all combinations of cerebellar recordings (Crus I-Lob II/III, $n=8$; Crus I-Lob VI, $n=7$; Lob II/III-Lob VI, $n=$ 675 7). 
A

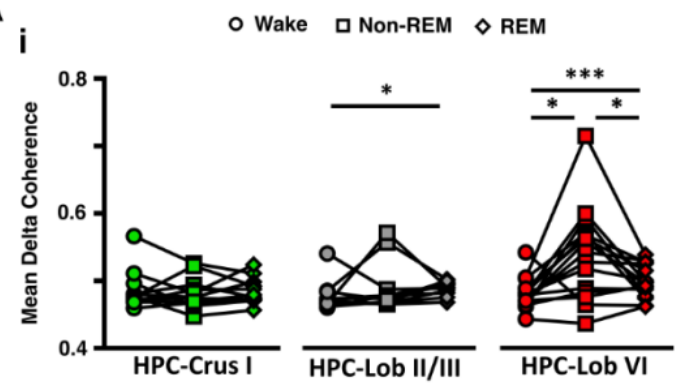

B i

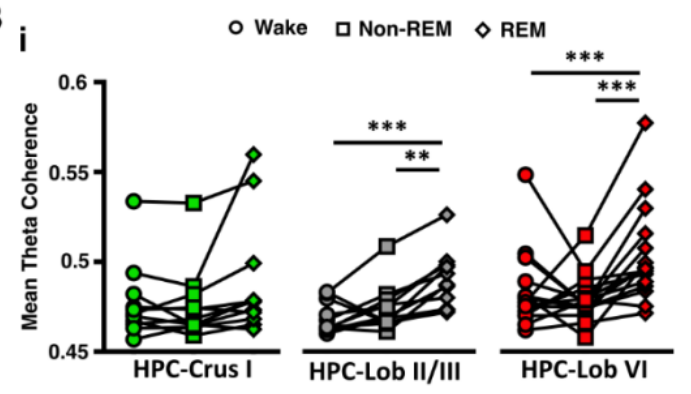

ii

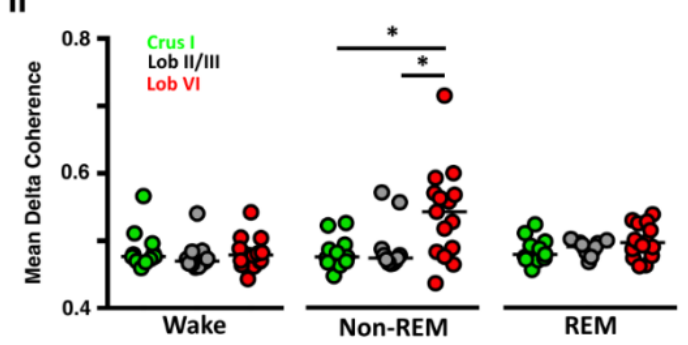

ii

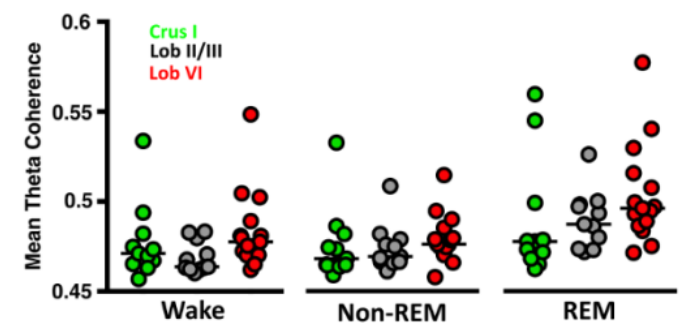

677 Supplemental Figure 3. Cerebello-hippocampal coherence in the delta ( $<4 \mathrm{~Hz})$ and theta $(6-12 \mathrm{~Hz})$

678 frequency ranges is modulated across sleep-states. A, i, Significant modulation across sleep states in

679 delta coherence was found between hippocampus and Lob II/III $(n=11$, Friedman test, $p=0.0273$,

680 multiple comparisons with FDR correction: wake vs non-REM $p=0.3657$, wake vs REM $p=0.0221$,

681 non-REM vs REM $p=0.0578)$, and particularly between the hippocampus and Lob VI $(n=15$, Friedman

682 test, $p=0.0006$, multiple comparisons with FDR correction: wake vs non-REM $p=0.0001$, wake vs

683 REM $p=0.0149$, non-REM vs REM $p=0.0351)$. Hippocampus-Crus I delta coherence remained

684 unchanged $(n=11$, Friedman test, $p=0.8438)$. ii, Significant differences in delta coherence between

685 the cerebello-hippocampal combinations were restricted to non-REM epochs, with HPC-Lob VI levels

686 being significantly higher than other combinations (wake, Kruskal-Wallis test, $p=0.4283$; non-REM,

687 Kruskal-Wallis test, $p=0.0225$, multiple comparisons with FDR correction: HPC-Crus I vs HPC-Lob II/III,

$688 p=0.3225$, HPC-Crus I vs HPC-Lob VI, $p=0.0122$, HPC-Lob II/III vs HPC-Lob VI, $p=0.0122$; REM, Kruskal-

689 Wallis test, $p=0.2826)$. B, i, Similarly, theta coherence was significantly increased during REM sleep

690 between HPC-Lob II/III $(n=11$, Friedman test, $\mathrm{p}=0.0002$, multiple comparisons with FDR correction:

691 wake vs non-REM $p=0.1378$, wake vs REM $p=0.0003$, non-REM vs REM $p=0.0029$ ) and HPC-Lob VI

692 ( $n=15$, Friedman test, $p=0.0007$, multiple comparisons with FDR correction: wake vs non-REM $p=$

6930.2503 , wake vs REM $p=0.001$, non-REM vs REM $p=0.0005)$, but remained stable across sleep states

694 between the HPC-Crus I ( $n=11$, Friedman test, $p=0.1632$ ). ii, In contrast, no differences across

695 cerebello-hipocampal combinations were found during any of the states analysed (wake, Kruskal-

696 Wallis test, $p=0.0677$; non-REM, Kruskal-Wallis test, $p=0.1994 ;$ REM, Kruskal-Wallis test, $p=0.0979$ ). 
A

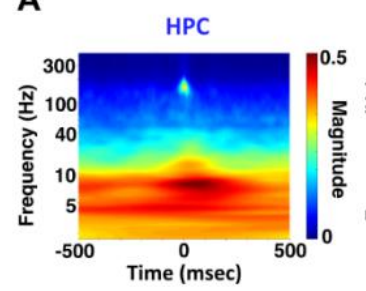

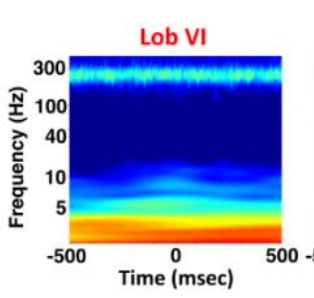

Lob II/III

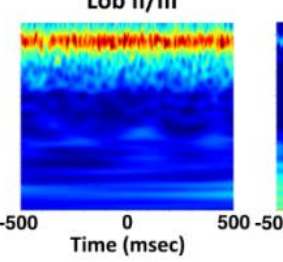

Crus I

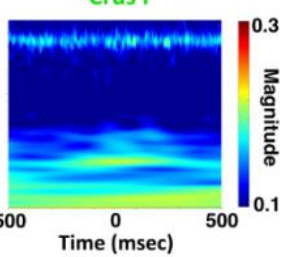

B Crus I Lob II/III Lob VI

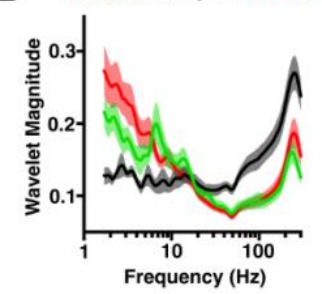

Supplemental Figure 4. SWR triggered spectral analysis. A, Averaged power spectra triggered by detected hippocampal SWR events (time zero). Nitzan et al., (2020) have recently shown that SWRs can propagate to the cortex. Here we observe discrete SWR activity in the hippocampal spectrogram $(\sim 150 \mathrm{~Hz})$ that is not present in the cerebellum. B, Mean cerebellar power spectra calculated during the 500ms following ripple detection.

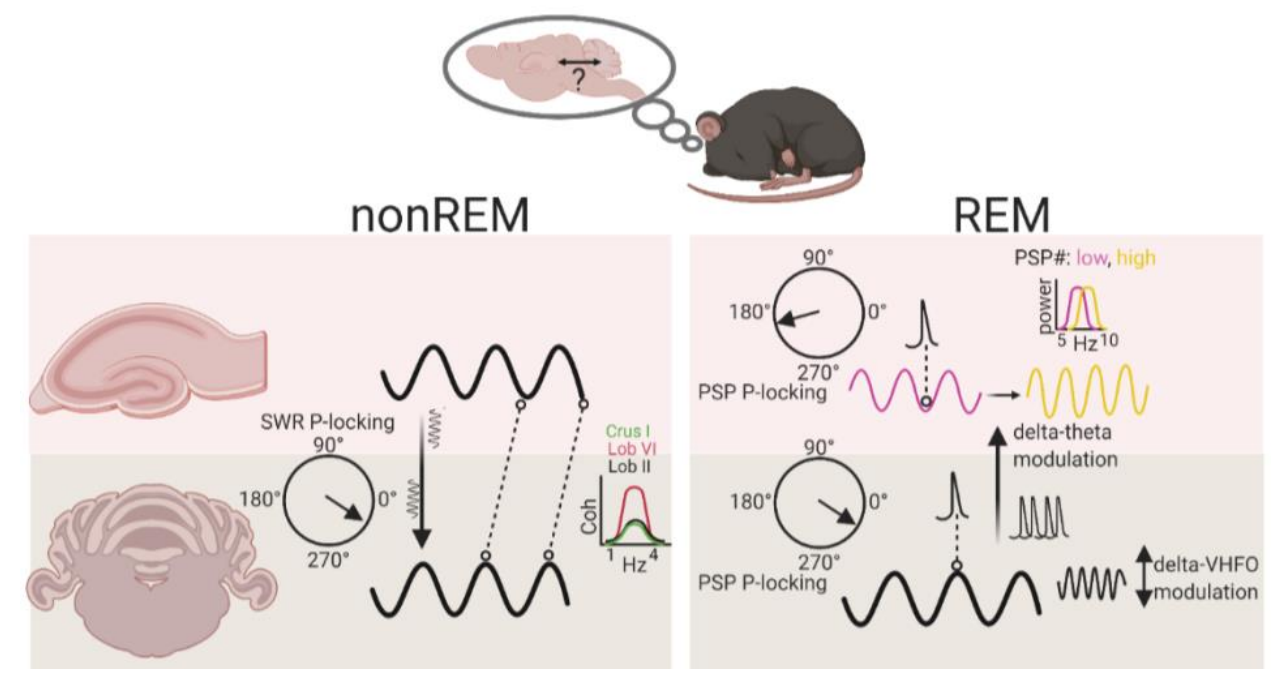

Supplemental Figure 5. Summary diagram of main findings illustrating sleep-stage specific physiological events and interactions within the cerebello-hippocampal network. During nonREM, coherence within the delta frequency range is highest between hippocampus and lobule VI compared to other cerebellar lobules. In addition, hippocampal sharp wave ripples (SWR) are phase locked to the cerebellar delta oscillation (black line) and drive modulation of cerebellar LFP activity. During REM, PSPs are significantly phase locked to both the trough of hippocampal theta (purple line) and the peak of cerebellar delta oscillations (black line). Within the cerebellum, delta oscillations can modulate activity within the VHFO range during REM. Additionally, cerebellar delta oscillations and associated PSPs modulate the frequency of hippocampal theta rhythms (from $~ 7.5 \mathrm{~Hz}$ (low, purple line) to $8 \mathrm{~Hz}$ (high, yellow line)). Abbreviations: SWR, sharp wave ripple; P-locking, phase locking; PSP, phasic sharp potential; VHFO, very high frequency oscillation. Created with BioRender.com. 
Babayan BM, Watilliaux A, Viejo G, Paradis A-L, Girard B, Rondi-Reig L. 2017. A hippocampocerebellar centred network for the learning and execution of sequence-based navigation. Sci Rep 7:17812. doi:10.1038/s41598-017-18004-7

Bernardi G, Betta M, Ricciardi E, Pietrini P, Tononi G, Siclari F. 2019. Regional delta waves in 726 human rapid eye movement sleep. I Neurosci 39:2686-2697. doi:10.1523/JNEUROSCI.2298-18.2019

Braun A. 1997. Regional cerebral blood flow throughout the sleep-wake cycle. An H2(15)O PET study. Brain 120:1173-1197. doi:10.1093/brain/120.7.1173

Burguière E, Arleo A, Hojjati M reza, Elgersma Y, Zeeuw Cl De, Berthoz A, Rondi-Reig L. 2005. Spatial navigation impairment in mice lacking cerebellar LTD: a motor adaptation deficit? Nat Neurosci 8:1292-1294. doi:10.1038/nn1532

Buzsáki G. 1986. Hippocampal sharp waves: Their origin and significance. Brain Res 398:242-252. doi:10.1016/0006-8993(86)91483-6

Canto CB, Onuki Y, Bruinsma B, van der Werf YD, De Zeeuw Cl. 2017. The Sleeping Cerebellum. Trends Neurosci. doi:10.1016/j.tins.2017.03.001

Cheron G, Gall D, Servais L, Dan B, Maex R, Schiffmann SN. 2004. Inactivation of Calcium-Binding Protein Genes Induces $160 \mathrm{~Hz}$ Oscillations in the Cerebellar Cortex of Alert Mice. J Neurosci 24:434-441. doi:10.1523/JNEUROSCI.3197-03.2004

Cheron G, Servais L, Wagstaff J, Dan B. 2005. Fast cerebellar oscillation associated with ataxia in a mouse model of angelman syndrome. Neuroscience 130:631-637. doi:10.1016/j.neuroscience.2004.09.013

Csicsvari J, Hirase H, Mamiya A, Buzsáki G. 2000. Ensemble patterns of hippocampal CA3-CA1 neurons during sharp wave-associated population events. Neuron 28:585-594. doi:10.1016/S0896-6273(00)00135-5

Datta S. 2000. Avoidance task training potentiates phasic pontine-wave density in the rat: A mechanism for sleep-dependent plasticity. J Neurosci 20:8607-8613. doi:10.1523/jneurosci.20-22-08607.2000

Datta S, O'Malley MW. 2013. Fear extinction memory consolidation requires potentiation of pontine-wave activity during REM sleep. I Neurosci 33:4561-4569. doi:10.1523/JNEUROSCI.5525-12.2013 
Datta S, Siwek DF, Patterson EH, Cipolloni PB. 1998. Localization of pontine PGO wave generation sites and their anatomical projections in the rat. Synapse 30:409-423. doi:10.1002/(sici)1098-2396(199812)30:4<409::aid-syn8>3.0.co;2-\%23

de Lavilléon G, Lacroix MM, Rondi-Reig L, Benchenane K. 2015. Explicit memory creation during sleep demonstrates a causal role of place cells in navigation. Nat Neurosci 18:493-495. doi:10.1038/nn.3970

de Solages C, Szapiro G, Brunel N, Hakim V, Isope P, Buisseret P, Rousseau C, Barbour B, Léna C. 2008. High-Frequency Organization and Synchrony of Activity in the Purkinje Cell Layer of the Cerebellum. Neuron 58:775-788. doi:10.1016/j.neuron.2008.05.008

De Zeeuw $\mathrm{Cl}$, Hoebeek FE, Schonewille M. 2008. Causes and consequences of oscillations in the cerebellar cortex. Neuron 58:655-8. doi:10.1016/j.neuron.2008.05.019

Diekelmann S, Born J. 2010. The memory function of sleep. Nat Rev Neurosci. doi:10.1038/nrn2762

Engbers JDT, Fernandez FR, Turner RW. 2013. Bistability in Purkinje neurons: Ups and downs in 766 cerebellar research. Neural Networks 47:18-31. doi:10.1016/j.neunet.2012.09.006

Farber J, Marks GA, Roffwarg HP. 1980. Rapid eye movement sleep PGO-type waves are present 768 in the dorsal pons of the albino rat. Science (80- ) 209:615-617. doi:10.1126/science.6994229

Franklin K, Paxinos G. 2007. The mouse brain in stereotaxic coordinates, 3rd ed. Elsevier.

Funk CM, Honjoh S, Rodriguez A V., Cirelli C, Tononi G. 2016. Local slow waves in superficial layers of primary cortical areas during REM sleep. Curr Biol 26:396-403. doi:10.1016/j.cub.2015.11.062

Girardeau G, Benchenane K, Wiener SI, Buzsáki G, Zugaro MB. 2009. Selective suppression of 775 hippocampal ripples impairs spatial memory. Nat Neurosci 12:1222-1223. doi:10.1038/nn.2384

Harlay F, Pellet J, Tardy MF, Dubrocard S. 1974. Activité unitaire corticocérébelleuse et mouvements oculaires: Modifications associées aux saccades du sommeil et de la veille. Physiol Behav 12:939-949. doi:10.1016/0031-9384(74)90141-3 sleep and waking. Electroencephalogr Clin Neurophysiol 33:457-469. doi:10.1016/0013- 

wake or not to wake? The two-sided nature of the human K-complex. Neuroimage 59:1631-1638. doi:10.1016/j.neuroimage.2011.09.013

Karashima A, Katayama N, Nakao M. 2007. Phase-locking of spontaneous and tone-elicited pontine waves to hippocampal theta waves during REM sleep in rats. Brain Res 1182:7381. doi:10.1016/j.brainres.2007.08.060

Karashima A, Nakamura K, Sato N, Nakao M, Katayama N, Yamamoto M. 2002. Phase-locking of spontaneous and elicited ponto-geniculo-occipital waves is associated with acceleration of hippocampal theta waves during rapid eye movement sleep in cats. Brain Res 958:347-358. doi:10.1016/S0006-8993(02)03673-9

Kaufmann C, Wehrle R, Wetter TC, Holsboer F, Auer DP, Pollmächer T, Czisch M. 2006. Brain activation and hypothalamic functional connectivity during human non-rapid eye movement sleep: An EEG/fMRI study. Brain 129:655-667. doi:10.1093/brain/awh686

Klinzing JG, Niethard N, Born J. 2019. Mechanisms of systems memory consolidation during sleep. Nat Neurosci. doi:10.1038/s41593-019-0467-3

Latchoumane CF V., Ngo HV V., Born J, Shin HS. 2017. Thalamic Spindles Promote Memory 799 Formation during Sleep through Triple Phase-Locking of Cortical, Thalamic, and Hippocampal Rhythms. Neuron 95:424-435.e6. doi:10.1016/j.neuron.2017.06.025 cerebellar Purkinje cell potentiation generates unstable spatial map orientation and inaccurate navigation. Nat Commun 10:1-13. doi:10.1038/s41467-019-09958-5 coupling mediates memory consolidation during sleep. Nat Neurosci 19:959-964. doi:10.1038/nn.4304

Mano NI. 1970. Changes of simple and complex spike activity of cerebellar purkinje cells with sleep and waking. Science (80- ) 170:1325-1327. doi:10.1126/science.170.3964.1325 sleep. Brain Res 17:145-148. doi:10.1016/0006-8993(70)90317-3 waves in the albino rat. Exp Neurol 69:648-666. doi:10.1016/0014-4886(80)90058-8 
McCarley RW, Hobson JA. 1972. Simple spike firing patterns of cat cerebellar purkinje cells in sleep and waking. Electroencephalogr Clin Neurophysiol 33:471-483. doi:10.1016/00134694(72)90211-8

Montgomery SM, Sirota A, Buzsáki G. 2008. Theta and gamma coordination of hippocampal networks during waking and rapid eye movement sleep. J Neurosci 28:6731-6741. doi:10.1523/JNEUROSCI.1227-08.2008

Newman PP, Reza H. 1979. Functional relationships between the hippocampus and the cerebellum: an electrophysiological study of the cat. J Physiol 287:405-26.

Nicola, Geva-Sagiv M, Nir Y. 2019. Local Sleep Oscillations: Implications for Memory Consolidation. Front Neurosci / www.frontiersin.org 1:813. doi:10.3389/fnins.2019.00813

Niedermeyer E, Uematsu S. 1974. Electroencephalographic recordings from deep cerebellar structures in patients with uncontrolled epileptic seizures. Electroencephalogr Clin Neurophysiol 37:355-365. doi:10.1016/0013-4694(74)90111-4

Nitzan N, McKenzie S, Beed P, English DF, Oldani S, Tukker JJ, Buzsáki G, Schmitz D. 2020. Propagation of hippocampal ripples to the neocortex by way of a subiculum-retrosplenial pathway. Nat Commun 11:1-17. doi:10.1038/s41467-020-15787-8

Palmer C. 1979. Interpositus and fastigial unit activity during sleep and waking in the cat. Electroencephalogr Clin Neurophysiol 46:357-370. doi:10.1016/0013-4694(79)90137-8 electrocerebellogram during sleep in the chronic cat. - PsycNET. Arch Ital Biol 115:108-13. involvement in cortical sensorimotor circuits for the control of voluntary movements. Nat Neurosci 17:1233-1239. doi:10.1038/nn.3773 Hippocampal Spatial Code. Science (80- ) 334:385-389. doi:10.1126/science.1207403 system. Front Neural Circuits 7:35. doi:10.3389/fncir.2013.00035 Neuronal Circuits in Cerebellar Cortex. J Neurosci 29. 
Roš H, Sachdev RNS, Yu Y, Šestan N, McCormick DA. 2009b. Neocortical networks entrain neuronal circuits in cerebellar cortex. J Neurosci 29:10309-10320. doi:10.1523/JNEUROSCI.2327-09.2009

Rowland NC, Goldberg JA, Jaeger D. 2010. Cortico-cerebellar coherence and causal connectivity during slow-wave activity.

Neuroscience 166:698-711. doi:10.1016/j.neuroscience.2009.12.048

Saint-Cyr J A, Woodward DJ. 1980. A topographic analysis of limbic and somatic inputs to the cerebellar cortex in the rat. Exp brain Res 40:13-22.

850

Saint-Cyr J.A., Woodward DJ. 1980b. Activation of mossy and climbing fiber pathways to the cerebellar cortex by stimulation of the fornix in the rat. Exp Brain Res 40:1-12. doi:10.1007/BF00236657

Schabus M, Dang-Vu TT, Albouy G, Balteau E, Boly M, Carrier J, Darsaud A, Degueldre C, Desseilles M, Gais S, Phillips C, Rauchs G, Schnakers C, Sterpenich V, Vandewalle G, Luxen A, Maquet P. 2007. Hemodynamic cerebral correlates of sleep spindles during human non-rapid eye movement sleep. Proc Natl Acad Sci U S A 104:13164-13169. doi:10.1073/pnas.0703084104

Schmahmann JD, Pandya DN. 1997. Anatomic organization of the basilar pontine projections 859 from prefrontal cortices in rhesus monkey. J Neurosci 17:438-458. doi:10.1523/jneurosci.17-01-00438.1997

Schwarz C. 2010. The fate of spontaneous synchronous rhythms on the cerebrocerebellar loop. Cerebellum 9:77-87. doi:10.1007/s12311-009-0143-3 Cheron G. 2005. Mono- and dual-frequency fast cerebellar oscillation in mice lacking parvalbumin and/or calbindin D-28k. Eur J Neurosci 22:861-870. doi:10.1111/j.14609568.2005.04275.x

Siclari F, Tononi G. 2017. Local aspects of sleep and wakefulness. Curr Opin Neurobiol. doi:10.1016/j.conb.2017.05.008 dynamics and their interactions with hippocampus across sleep states. Elife 9. doi:10.7554/eLife. 52244 
872

873

874

875

876

877

878

879

880

881

882

883

884

885

886

Velluti R, Yamuy J, Hadjez J, Monti JM. 1985. Spontaneous cerebellar nuclei PGO-like waves in natural paradoxical sleep and under reserpine. Electroencephalogr Clin Neurophysiol 60:243-248. doi:10.1016/0013-4694(85)90038-0

Wang D V., Ikemoto S. 2016. Coordinated interaction between hippocampal sharp-wave ripples and anterior cingulate unit activity. J Neurosci 36:10663-10672. doi:10.1523/JNEUROSCI.1042-16.2016

Watson TC, Obiang P, Torres-Herraez A, Watilliaux A, Coulon P, Rochefort C, Rondi-Reig L. 2019. Anatomical and physiological foundations of cerebello-hippocampal interaction. Elife 8. doi:10.7554/eLife.41896.001

Xu W, De Carvalho F, Clarke AK, Jackson A. 2020. Communication from the cerebellum to the neocortex during sleep spindles. Prog Neurobiol. doi:10.1016/j.pneurobio.2020.101940

Zhang L Bin, Zhang J, Sun MJ, Chen H, Yan J, Luo FL, Yao ZX, Wu YM, Hu B. 2020. Neuronal Activity in the Cerebellum During the Sleep-Wakefulness Transition in Mice. Neurosci Bull 36:919931. doi:10.1007/s12264-020-00511-9 